Supplement of Biogeosciences, 12, 3429-3446, 2015

http://www.biogeosciences.net/12/3429/2015/

doi:10.5194/bg-12-3429-2015-supplement

(C) Author(s) 2015. CC Attribution 3.0 License.

(c) (i)

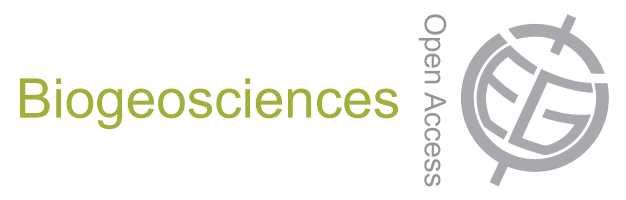

Supplement of

\title{
Estimates of micro-, nano-, and picoplankton contributions to particle ex- port in the northeast Pacific
}

\section{B. L. Mackinson et al.}

Correspondence to: B. L. Mackinson (bmackinson@my.uri.edu)

The copyright of individual parts of the supplement might differ from the CC-BY 3.0 licence. 
Table S1: POC, $\mathrm{Chl} a$, and indicator pigment concentrations from small volume samples from the photic zone. Whole Chl $a$ and $>5 \mu \mathrm{m} \mathrm{Chl} a$ are determined by fluorescence while Chl $a$ and indicator pigment concentrations are measured with HPLC.

\begin{tabular}{|c|c|c|c|c|c|c|c|c|c|c|c|}
\hline $\begin{array}{l}\text { Depth } \\
(\mathrm{m})\end{array}$ & $\begin{array}{c}\text { POC } \\
\left(\mu \mathrm{mol} \mathrm{L}{ }^{-1}\right)\end{array}$ & $\begin{array}{c}\text { Whole Chl } a \\
\left(\text { ng L L }^{-1}\right)\end{array}$ & $\begin{array}{c}>5 \text { um Chl } a \\
\left(\mathrm{ng} \mathrm{L}^{-1}\right)\end{array}$ & $\begin{array}{c}\text { Chl } a \\
\left(\operatorname{ng~L}^{-1}\right)\end{array}$ & $\begin{array}{l}\text { FUCO } \\
\left(\mathrm{ng} \mathrm{L}^{-1}\right)\end{array}$ & $\begin{array}{c}\text { PER } \\
\left(\operatorname{ng~L}^{-1}\right)\end{array}$ & $\begin{array}{c}\text { HEX } \\
\left(n g L^{-1}\right)\end{array}$ & $\begin{array}{c}\text { BUT } \\
\left(\operatorname{ng~L}^{-1}\right)\end{array}$ & $\begin{array}{l}\text { Allo } \\
\left(\text { ng L }^{-1}\right)\end{array}$ & $\begin{array}{c}\text { Chl } b \\
\left(\mathrm{ng} \mathrm{L}^{-1}\right)\end{array}$ & $\begin{array}{l}\text { ZEA } \\
\left(\mathrm{ng} \mathrm{L}^{-1}\right)\end{array}$ \\
\hline \multicolumn{12}{|c|}{ Aug. 2010 P4 } \\
\hline 5 & - & 1962.06 & 572.94 & - & - & - & - & - & - & - & - \\
\hline 10 & - & 1594.51 & 470.25 & - & - & - & - & - & - & - & - \\
\hline 20 & - & 1940.44 & 875.63 & - & - & - & - & - & - & - & - \\
\hline 21 & - & 1691.80 & 916.17 & - & - & - & - & - & - & - & - \\
\hline 30 & - & 68.64 & 92.43 & - & - & - & - & - & - & - & - \\
\hline 50 & - & 38.92 & 21.62 & - & - & - & - & - & - & - & - \\
\hline 75 & - & 32.97 & 25.40 & - & - & - & - & - & - & - & - \\
\hline \multicolumn{12}{|c|}{ Aug. 2010 P12 } \\
\hline 5 & - & 400.65 & 85.81 & - & - & - & - & - & - & - & - \\
\hline 10 & - & 307.42 & 95.27 & 358.00 & 39.00 & 7.00 & 60.00 & 23.00 & 4.00 & 40.00 & 48.00 \\
\hline 20 & - & 452.68 & 160.80 & 541.00 & 73.00 & 7.00 & 84.00 & 26.00 & 4.00 & 74.00 & 76.00 \\
\hline 27 & - & 513.49 & 290.19 & - & - & - & - & - & - & - & - \\
\hline 30 & - & 486.46 & 232.42 & 558.00 & 87.00 & 9.00 & 215.00 & 23.00 & 5.00 & 102.00 & 15.00 \\
\hline 50 & - & 142.56 & 37.16 & - & - & - & - & - & - & - & - \\
\hline 75 & - & 59.46 & 17.50 & 61.00 & 8.00 & 1.00 & 29.00 & 7.00 & 1.00 & 13.00 & 1.00 \\
\hline \multicolumn{12}{|c|}{ Aug. 2010 P16 } \\
\hline 5 & - & 172.63 & 85.81 & - & - & - & - & - & - & - & - \\
\hline 10 & - & 192.56 & 84.12 & 179.00 & 15.00 & 9.00 & 54.00 & 15.00 & 1.00 & 27.00 & 1.00 \\
\hline 20 & - & 212.15 & 88.85 & - & - & - & - & - & - & - & - \\
\hline 30 & - & 283.09 & 116.21 & 270.00 & 26.00 & 7.00 & 124.00 & 17.00 & 1.00 & 54.00 & 2.00 \\
\hline 40 & - & 436.13 & 93.91 & - & - & - & - & - & - & - & - \\
\hline 50 & - & 187.15 & 55.40 & - & - & - & - & - & - & - & - \\
\hline 50 & - & 142.56 & 60.13 & - & - & - & - & - & - & - & - \\
\hline 50 & - & 164.86 & 57.77 & - & - & - & - & - & - & - & - \\
\hline 75 & - & 170.60 & 29.42 & 160.00 & 13.00 & 1.00 & 64.00 & 14.00 & 1.00 & 25.00 & 4.00 \\
\hline \multicolumn{12}{|c|}{ Aug. 2010 P20 } \\
\hline 5 & - & 271.61 & 50.00 & - & - & - & - & - & - & - & - \\
\hline 10 & - & 193.23 & 61.48 & 213.00 & 21.00 & 5.00 & 82.00 & 18.00 & 1.00 & 25.00 & 1.00 \\
\hline 20 & - & 279.71 & 72.97 & - & - & - & - & - & - & - & - \\
\hline 30 & - & 493.22 & 158.10 & 386.00 & 79.00 & 10.00 & 118.00 & 26.00 & 1.00 & 48.00 & 2.00 \\
\hline 36 & - & 439.17 & 167.56 & - & - & - & - & - & - & - & - \\
\hline 36 & - & 466.19 & 135.13 & - & - & - & - & - & - & - & - \\
\hline 50 & - & 362.82 & 164.86 & - & - & - & - & - & - & - & - \\
\hline 75 & - & 172.96 & 34.05 & 194.00 & 16.00 & 1.00 & 73.00 & 19.00 & 1.00 & 36.00 & 5.00 \\
\hline
\end{tabular}

Aug. 2010 P26

$\begin{array}{cccc}5 & - & 226.00 & 104.39 \\ 10 & - & 230.06 & 111.48 \\ 20 & - & 237.49 & 115.53 \\ 30 & - & 306.06 & 363.83 \\ 35 & - & 334.44 & 156.07 \\ 50 & - & 302.01 & 91.89 \\ 75 & - & 118.57 & 36.48\end{array}$

Feb. 2011 P12

$\begin{array}{cccccccccccc}1 & - & 271.88 & 60.42 & 550.00 & 50.00 & 6.00 & 102.00 & 45.00 & 14.00 & 131.00 & 17.00 \\ 35 & - & 324.74 & 45.31 & - & - & - & - & - & - & - & - \\ 43 & - & 286.98 & 41.54 & 431.00 & 42.00 & 6.00 & 82.00 & 35.00 & 13.00 & 102.00 & 13.00\end{array}$




$\begin{array}{rccccccccccc}48 & - & 313.41 & 41.54 & - & - & - & - & - & - & - & - \\ 55 & - & 313.41 & 56.64 & 445.00 & 44.00 & 6.00 & 84.00 & 36.00 & 13.00 & 103.00 & 14.00 \\ 60 & - & 302.09 & 41.54 & - & - & - & - & - & - & - & - \\ 65 & - & 290.76 & 52.87 & 408.00 & 36.00 & 5.00 & 70.00 & 29.00 & 10.00 & 99.00 & 12.00\end{array}$

Feb. 2011 P16

1
7
13
20
40
95

Feb. 2011 P20

1
6
11
19
38
50
77

156.08
156.08
143.49
168.66
191.32
173.70
138.46

June 2011 P4

1
4
9
17
25
38
72

254.89
237.14
243.93
181.25
394.22
335.32
341.73

15.10
12.59
15.10
15.10
20.14
17.62
12.59

$\begin{array}{cccccccc}236.00 & 19.00 & 2.00 & 84.00 & 25.00 & 2.00 & 62.00 & 24.00 \\ - & - & - & - & - & - & - & - \\ 231.00 & 18.00 & 2.00 & 80.00 & 24.00 & 2.00 & 62.00 & 25.00 \\ - & - & - & - & - & - & - & - \\ 234.00 & 18.00 & 3.00 & 84.00 & 25.00 & 2.00 & 63.00 & 24.00 \\ 26.00 & 3.00 & 1.00 & 8.00 & 3.00 & 1.00 & 10.00 & 1.00\end{array}$

June 2011 P12

1
5
15
20
40
65
90

$$
\begin{aligned}
& 262.44 \\
& 255.26 \\
& 287.93 \\
& 250.73 \\
& 257.61 \\
& 228.07 \\
& 141.98
\end{aligned}
$$
17.37
25.68
17.37
12.08
28.70
13.59
19.22

$\begin{array}{cccccccc}828.00 & 79.00 & 14.00 & 265.00 & 104.00 & 19.00 & 160.00 & 76.00 \\ - & - & - & - & - & - & - & - \\ 774.00 & 69.00 & 11.00 & 231.00 & 89.00 & 16.00 & 150.00 & 66.00 \\ - & - & - & - & - & - & - & - \\ 804.00 & 77.00 & 12.00 & 269.00 & 103.00 & 19.00 & 159.00 & 70.00 \\ - & - & - & - & - & - & - & - \\ 516.00 & 51.00 & 1.00 & 184.00 & 58.00 & 5.00 & 134.00 & 31.00\end{array}$
$516.00-51.00$

$\begin{array}{cccccccc}340.00 & 33.00 & 6.00 & 78.00 & 18.00 & 21.00 & 33.00 & 58.00 \\ - & - & - & - & - & - & - & - \\ 233.00 & 22.00 & 4.00 & 53.00 & 13.00 & 13.00 & 24.00 & 44.00 \\ - & - & - & - & - & - & - & - \\ 496.00 & 39.00 & 1.00 & 169.00 & 37.00 & 10.00 & 110.00 & 98.00 \\ - & - & - & - & - & - & - & - \\ 426.00 & 43.00 & 1.00 & 185.00 & 54.00 & 7.00 & 83.00 & 59.00\end{array}$

June 2011 P16

21.90

21.15

18.13

21.15

20.77

24.17

14.10

$\begin{array}{cccccccc}- & - & - & - & - & - & - & - \\ 391.00 & 51.00 & 1.00 & 134.00 & 27.00 & 13.00 & 44.00 & 108.00 \\ 387.00 & 51.00 & 1.00 & 136.00 & 28.00 & 14.00 & 39.00 & 97.00 \\ - & - & - & - & - & - & - & - \\ 339.00 & 75.00 & 1.00 & 203.00 & 45.00 & 3.00 & 25.00 & 20.00 \\ - & - & - & - & - & - & - & - \\ 187.00 & 44.00 & 1.00 & 53.00 & 38.00 & 3.00 & 33.00 & 3.00\end{array}$

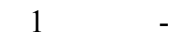

12 -

26

199.50

52.87

$220.00 \quad 46.00$

43.80

49.84

149.36

232.61

70.99

173.91

28.70

193.34

49.84

140.76

5.29

June 2011 P20

$\begin{array}{cccc}1 & - & 149.36 & 27.19 \\ 8 & - & 145.00 & 28.70 \\ 15 & - & 149.99 & 29.45 \\ 22 & - & 234.12 & 40.78 \\ 34 & - & 312.78 & 59.66 \\ 50 & - & 330.98 & 77.45 \\ 70 & - & 155.57 & 18.13\end{array}$

27.19
28.70
29.45
40.78
59.66
77.45
18.13

$\begin{array}{cccccccc}220.00 & 46.00 & 1.00 & 106.00 & 15.00 & 3.00 & 9.00 & 13.00 \\ - & - & - & - & - & - & - & - \\ 214.00 & 48.00 & 1.00 & 106.00 & 15.00 & 2.00 & 9.00 & 14.00 \\ - & - & - & - & - & - & - & - \\ 237.00 & 42.00 & 1.00 & 150.00 & 23.00 & 1.00 & 26.00 & 24.00 \\ - & - & - & - & - & - & - & - \\ 167.00 & 20.00 & 1.00 & 50.00 & 28.00 & 2.00 & 50.00 & 3.00\end{array}$

$\begin{array}{cccccccc}199.50 & 35.50 & 3.50 & 86.50 & 15.50 & 2.00 & 10.00 & 16.00 \\ - & - & - & - & - & - & - & - \\ 199.00 & 47.00 & 1.00 & 75.00 & 16.00 & 2.00 & 11.00 & 14.00 \\ - & - & - & - & - & - & - & - \\ 394.00 & 102.00 & 5.00 & 194.00 & 39.00 & 3.00 & 23.00 & 24.00 \\ - & - & - & - & - & - & - & - \\ 217.00 & 33.00 & 1.00 & 53.00 & 26.00 & 2.00 & 67.00 & 7.00\end{array}$


June 2011 P26

$\begin{array}{cccccccccccc}1 & - & 178.21 & 30.21 & 239.00 & 47.00 & 3.00 & 59.00 & 30.00 & 2.00 & 24.00 & 45.00 \\ 5 & - & 179.74 & 30.21 & 224.78 & 45.79 & - & 66.68 & 28.57 & 4.02 & 23.74 & 41.67 \\ 9 & - & 173.49 & 30.96 & 230.30 & 45.62 & 0.50 & 62.22 & 27.86 & 1.00 & 22.04 & 43.75 \\ 16 & - & 178.23 & 34.74 & 235.21 & 48.66 & - & 69.45 & 31.57 & 3.26 & 23.74 & 45.11 \\ 24 & - & 259.92 & 40.78 & 339.97 & 77.28 & 0.50 & 104.28 & 48.46 & 4.47 & 37.79 & 51.70 \\ 52 & - & 394.29 & 86.09 & 489.71 & 114.16 & - & 212.17 & 82.63 & 2.15 & 60.83 & 40.41 \\ 83 & - & 152.55 & 36.25 & 225.00 & 45.00 & 7.00 & 54.00 & 29.00 & 2.00 & 51.00 & 9.00\end{array}$

June 2011 P26

1
5
10
15
24
48
85

P26
-
-
-
-
-
-

$\begin{array}{cc}221.53 & 33.98 \\ 225.05 & 42.29 \\ 220.59 & 39.27 \\ 232.61 & 78.54 \\ 233.07 & 56.64 \\ 444.32 & 92.89 \\ 86.09 & 77.03\end{array}$

$\begin{array}{cc}282.39 & 62.03 \\ 278.37 & 74.44 \\ 272.35 & 71.66 \\ 286.71 & 76.50 \\ 295.00 & 81.50 \\ 522.81 & 152.93 \\ 115.79 & 26.95\end{array}$

$\begin{array}{cccccc}- & 90.93 & 26.45 & 3.24 & 21.36 & 28.07 \\ - & 99.85 & 33.01 & 5.07 & 23.24 & 35.35 \\ - & 96.23 & 32.87 & - & 20.89 & 33.42 \\ - & 103.13 & 33.34 & 4.48 & 24.43 & 35.11 \\ - & 105.00 & 35.00 & 5.00 & 27.00 & 36.00 \\ 4.86 & 198.44 & 87.58 & 2.08 & 63.10 & 25.72 \\ - & 37.44 & 27.51 & - & 15.46 & 4.68\end{array}$

Feb. 2012 P4

$\begin{array}{cccc}1 & - & - & - \\ 5 & 6.67 & 417.84 & 97.42 \\ 8 & - & 427.45 & 90.63 \\ 14 & 5.06 & 490.60 & 89.87 \\ 22 & 4.13 & 429.09 & 94.40 \\ 35 & - & 438.02 & 54.38 \\ 50 & 4.78 & 438.97 & 86.85\end{array}$

$\begin{array}{cccccccc}580.80 & 104.92 & - & 125.80 & 40.07 & 23.71 & 93.35 & 5.67 \\ - & - & - & - & - & - & - & - \\ 546.93 & 101.62 & - & 113.34 & 40.77 & 27.97 & 92.91 & - \\ 628.22 & 109.05 & - & 115.50 & 38.07 & 26.12 & 94.47 & 7.45 \\ 608.17 & 99.56 & - & 119.31 & 40.15 & 22.16 & 105.10 & 11.33 \\ 664.89 & 96.79 & - & 144.08 & 36.00 & 24.62 & 115.39 & 10.36 \\ 657.72 & 98.16 & - & 122.81 & 33.76 & 25.27 & 107.84 & 8.48\end{array}$

Feb. 2012 P12

$\begin{array}{cccccccccccc}1 & 2.76 & 12.08 & 36.75 & 276.66 & 38.51 & 6.82 & 147.38 & 50.76 & - & 46.91 & 11.99 \\ 8 & - & 212.97 & 31.72 & 299.87 & 38.72 & 10.12 & 155.20 & 51.76 & 3.41 & 52.30 & 12.01 \\ 12 & 2.58 & 208.44 & 30.96 & 282.59 & 37.14 & 4.09 & 147.06 & 48.29 & - & 53.60 & 12.73 \\ 26 & - & 244.69 & 36.25 & 314.93 & 40.21 & - & 156.79 & 53.89 & - & 52.44 & 14.96 \\ 41 & 2.80 & 222.03 & 33.98 & 283.90 & 35.96 & - & 143.26 & 47.78 & - & 49.67 & 13.05 \\ 55 & - & 237.14 & 37.76 & 241.39 & 32.62 & - & 118.89 & 39.82 & - & 33.14 & 8.04 \\ 95 & 1.66 & 57.40 & 14.35 & 74.36 & 15.80 & - & 29.70 & 11.24 & - & 18.05 & 4.96\end{array}$

Feb. 2012 P16

$\begin{array}{cccc}2 & 2.46 & 238.65 & 61.12 \\ 10 & - & 279.43 & 64.95 \\ 20 & 3.38 & 253.00 & 49.09 \\ 50 & - & 252.24 & 57.40 \\ 58 & 2.41 & 268.86 & 61.93 \\ 70 & - & 255.26 & 66.46 \\ 95 & 1.25 & 43.05 & 30.96\end{array}$

Feb. 2012 P20

$\begin{array}{cccc}1 & - & 297.55 & 90.25 \\ 10 & - & 234.12 & 83.07 \\ 30 & - & 304.35 & 80.05 \\ 50 & - & 454.64 & 71.75 \\ 75 & - & 267.35 & 72.50 \\ 100 & - & 164.64 & 55.13\end{array}$

Feb. 2012 P26

$\begin{array}{cccccccccccc}1 & 3.11 & 222.79 & 77.79 & 336.21 & 58.81 & 10.91 & 79.84 & 43.35 & 3.96 & 31.90 & 6.82 \\ 10 & - & 253.75 & 87.60 & 315.33 & 53.39 & 9.49 & 77.25 & 42.68 & 0.00 & 24.69 & 5.14 \\ 15 & 3.54 & 226.56 & 77.79 & 314.67 & 52.78 & 8.88 & 75.99 & 43.68 & 3.93 & 26.07 & 4.25 \\ 25 & - & 228.07 & 107.24 & 329.06 & 60.31 & 90.71 & 79.81 & 40.95 & 4.88 & 20.72 & 8.60\end{array}$




$\begin{array}{cccccccccccc}36 & 4.29 & 237.14 & 81.56 & 350.69 & 57.24 & 10.61 & 82.36 & 47.77 & 4.59 & 22.14 & 5.37 \\ 47 & - & 225.05 & 101.20 & 319.36 & 58.35 & 8.68 & 75.02 & 47.01 & - & 35.66 & 3.97 \\ 75 & 4.02 & 253.00 & 72.50 & 348.51 & 56.69 & 9.24 & 84.26 & 46.10 & - & 34.60 & 3.63\end{array}$

June 2012 P4

\begin{tabular}{|c|c|c|c|c|c|c|c|c|c|c|}
\hline 2 & 4.33 & 312.66 & 57.40 & 260.07 & 74.44 & - & 25.17 & - & - & - \\
\hline 13 & - & 306.62 & 120.83 & & & - & & - & - & - \\
\hline 18 & 7.07 & 220.52 & 194.85 & 348.55 & 125.29 & - & 33.15 & - & - & - \\
\hline 25 & - & 196.36 & 120.83 & & & - & & - & - & - \\
\hline 31 & 5.11 & 107.24 & 48.33 & 277.78 & 205.43 & - & 32.82 & - & - & - \\
\hline 71 & - & 128.55 & 78.54 & & & - & - & - & - & - \\
\hline 103 & 2.76 & 72.50 & 49.84 & 65.91 & 39.58 & - & - & - & - & - \\
\hline
\end{tabular}

June 2012 P12

$\begin{array}{ll}6 & 4.06\end{array}$

75 -

$111 \quad 2.50$

220.00

50.00

20.00

230.00

30.00

60.00

$\begin{array}{ccc}138 & - & 280.00 \\ 151 & 1.97 & 330.00\end{array}$

30.00

$158 \quad-\quad 170.00$

50.00

$\begin{array}{lll}164 & 1.10 & 130.00\end{array}$

20.00

$\begin{array}{cc}94.11 & 17.42 \\ 110.91 & 16.77 \\ - & - \\ - & - \\ - & - \\ 527.34 & 5.56\end{array}$

June 2012 P16

$5 \quad 5.15$

220.00

50.00

$142.92 \quad 26.97$

130.00

20.00

130.00

260.00

280.00

160.00

$\begin{array}{ll}16 & 5.67 \\ 25 & \end{array}$

$35 \quad 5.15$

330.00

30.00

50.00

$\begin{array}{ll}43 & - \\ 66 & 2.78\end{array}$

130.00

20.00

$\begin{array}{cc}142.92 & 26.97 \\ - & - \\ 188.11 & 37.67 \\ - & - \\ 317.23 & 87.74 \\ - & - \\ 77.70 & 35.58\end{array}$

$\begin{array}{ccccc}92.53 & 18.59 & - & - & 15.40 \\ - & - & - & - & - \\ 96.80 & 20.46 & - & 25.13 & 15.59 \\ - & - & - & - & - \\ 171.55 & 41.02 & - & - & - \\ - & - & - & - & - \\ 41.25 & 16.11 & - & - & -\end{array}$

June 2012 P20

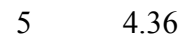

110.00

10.00

$451.86 \quad 5.56$

$82.99-$

182.76

$15 \quad-$

190.00

6.04

10.00

60.00

130.00

10.00

$\begin{array}{ccc}75 & - & 130.00 \\ 115 & 1.91 & 110.00\end{array}$

10.00

10.00

$\begin{array}{cc}- & - \\ 136.70 & 74.47\end{array}$

39. 24

- $\quad-$

$198.88 \quad 125.34$

$\begin{array}{cc}- & - \\ 26.81 & 205.51\end{array}$

$\begin{array}{ccc}- & 55.18 & 13.54 \\ - & 76.97 & 24.17 \\ - & - & - \\ - & - & - \\ - & - & - \\ - & - & -\end{array}$

June 2012 P26

$5 \quad 5.21$

$\begin{array}{ll}280.00 & 50.00 \\ 230.00 & 30.00 \\ 230.00 & 40.00 \\ 240.00 & 40.00 \\ 220.00 & 40.00 \\ 200.00 & 20.00 \\ 140.00 & 20.00\end{array}$

\begin{tabular}{cc}
254.86 & 35.60 \\
- & - \\
253.79 & 17.43 \\
- & - \\
290.59 & 16.78 \\
- & - \\
198.98 & - \\
\hline
\end{tabular}

\begin{tabular}{ccccc}
68.20 & 16.11 & - & 178.39 & 18.67 \\
- & - & - & - & - \\
97.35 & 13.54 & - & - & 18.37 \\
- & - & - & - & - \\
109.79 & 24.17 & - & 27.18 & 23.23 \\
- & - & - & - & - \\
232.74 & - & - & 86.43 & 43.46 \\
\hline
\end{tabular}






Table S2: Size-fractionated particulate ${ }^{234} \mathrm{Th}$ and POC concentrations

\begin{tabular}{|c|c|c|c|c|c|c|c|c|c|}
\hline $\begin{array}{l}\text { Depth } \\
\text { (m) }\end{array}$ & $\begin{array}{c}>53 \mu \mathrm{m}^{234} \mathrm{Th} \\
\left.(\mathrm{dpm} \mathrm{L})^{-1}\right)\end{array}$ & $\begin{array}{c}10-53 \mu \mathrm{m}^{234} \mathrm{Th} \\
\left(\mathrm{dpm} \mathrm{L} \mathrm{L}^{-1}\right)\end{array}$ & $\begin{array}{c}1-10 \mu \mathrm{m}^{234} \mathrm{Th} \\
\left(\mathrm{dpm} \mathrm{L} \mathrm{L}^{-1}\right)\end{array}$ & $\begin{array}{c}>53 \mu \mathrm{m} \text { POC } \\
\left.(\mu \mathrm{mol} \mathrm{L})^{-1}\right)\end{array}$ & $\begin{array}{c}10-53 \mu \mathrm{m} \text { POC } \\
\left.(\mu \mathrm{mol} \mathrm{L})^{-1}\right)\end{array}$ & $\begin{array}{c}1-10 \mu \mathrm{m} \text { POC } \\
\left(\mu \mathrm{mol} \mathrm{L}{ }^{-1}\right)\end{array}$ & $\begin{array}{c}>53 \mu \mathrm{m} \\
\mathrm{POC}{ }^{234} \mathrm{Th} \\
\left(\mu \mathrm{mol} \mathrm{dpm}{ }^{-1}\right)\end{array}$ & $\begin{array}{c}10-53 \mu \mathrm{m} \\
\mathrm{POC}{ }^{234} \mathrm{Th} \\
\left(\mu \mathrm{mol} \mathrm{dpm}{ }^{-1}\right)\end{array}$ & $\begin{array}{c}1-10 \mu \mathrm{m} \\
\mathrm{POC}{ }^{234} \mathrm{Th} \\
\left(\mu \mathrm{mol} \mathrm{dpm}{ }^{-1}\right)\end{array}$ \\
\hline \multicolumn{10}{|c|}{ June 2011 P26 } \\
\hline 30 & $0.106 \pm 0.005$ & $0.046 \pm 0.003$ & $0.047 \pm 0.003$ & $0.535 \pm 0.027$ & $0.191 \pm 0.010$ & $0.159 \pm 0.008$ & $5.0 \pm 0.4$ & $4.1 \pm 0.4$ & $3.4 \pm 0.3$ \\
\hline 50 & $0.046 \pm 0.002$ & $0.050 \pm 0.003$ & $0.032 \pm 0.002$ & $0.139 \pm 0.007$ & $0.118 \pm 0.006$ & $0.061 \pm 0.003$ & $3.0 \pm 0.2$ & $2.4 \pm 0.2$ & $1.9 \pm 0.2$ \\
\hline 100 & $0.087 \pm 0.005$ & $0.066 \pm 0.004$ & - & $0.129 \pm 0.007$ & $0.066 \pm 0.005$ & - & $1.5 \pm 0.1$ & $1.0 \pm 0.1$ & - \\
\hline 150 & $0.049 \pm 0.004$ & $0.025 \pm 0.002$ & $0.051 \pm 0.004$ & $0.116 \pm 0.006$ & $0.023 \pm 0.001$ & $0.065 \pm 0.004$ & $2.4 \pm 0.2$ & $0.9 \pm 0.1$ & $1.3 \pm 0.1$ \\
\hline 200 & $0.020 \pm 0.001$ & $0.050 \pm 0.003$ & $0.029 \pm 0.002$ & $0.026 \pm 0.001$ & $0.056 \pm 0.003$ & $0.017 \pm 0.001$ & $1.3 \pm 0.1$ & $1.1 \pm 0.1$ & $0.6 \pm 0.0$ \\
\hline
\end{tabular}

\section{Feb. 2012 P4}

$\begin{array}{ccccccccccc}30 & 0.007 \pm 0.001 & 0.047 \pm 0.004 & 0.045 \pm 0.003 & 0.028 \pm 0.002 & 0.183 \pm 0.009 & 0.135 \pm 0.007 & 3.9 \pm 0.5 & 3.9 \pm 0.4 & 3.0 \pm 0.3 \\ 50 & 0.003 \pm 0.000 & 0.019 \pm 0.002 & 0.016 \pm 0.001 & 0.039 \pm 0.002 & 0.103 \pm 0.005 & 0.077 \pm 0.004 & 12.5 \pm 1.7 & 5.5 \pm 0.5 & 4.8 \pm 0.5 \\ 100 & 0.016 \pm 0.001 & 0.079 \pm 0.005 & 0.048 \pm 0.004 & 0.037 \pm 0.002 & 0.135 \pm 0.007 & 0.060 \pm 0.003 & 2.3 \pm 0.3 & 1.7 \pm 0.1 & 1.2 \pm 0.1 \\ 150 & 0.024 \pm 0.002 & 0.080 \pm 0.006 & 0.022 \pm 0.002 & 0.042 \pm 0.002 & 0.103 \pm 0.005 & 0.036 \pm 0.002 & 1.8 \pm 0.2 & 1.3 \pm 0.1 & 1.7 \pm 0.2\end{array}$

Feb. 2012 P12

$\begin{array}{cccccccccc}30 & 0.009 \pm 0.001 & 0.046 \pm 0.004 & 0.023 \pm 0.002 & 0.036 \pm 0.002 & 0.184 \pm 0.009 & 0.081 \pm 0.004 & 4.1 \pm 0.5 & 4.0 \pm 0.4 & 3.5 \pm 0.4 \\ 50 & 0.038 \pm 0.003 & 0.040 \pm 0.004 & 0.028 \pm 0.003 & 0.101 \pm 0.005 & 0.155 \pm 0.008 & 0.086 \pm 0.004 & 2.6 \pm 0.3 & 3.9 \pm 0.4 & 3.0 \pm 0.3 \\ 100 & 0.036 \pm 0.005 & 0.033 \pm 0.004 & 0.022 \pm 0.003 & 0.228 \pm 0.013 & 0.265 \pm 0.014 & 0.261 \pm 0.014 & 6.3 \pm 0.9 & 7.9 \pm 1.1 & 11.6 \pm 1.5 \\ 200 & 0.013 \pm 0.001 & 0.066 \pm 0.005 & 0.026 \pm 0.003 & 0.037 \pm 0.002 & 0.098 \pm 0.005 & 0.042 \pm 0.002 & 2.8 \pm 0.3 & 1.5 \pm 0.1 & 1.6 \pm 0.2\end{array}$

Feb. 2012 P26

$\begin{array}{cccccccccccc}30 & 0.118 \pm 0.014 & 0.221 \pm 0.028 & 0.120 \pm 0.015 & 1.836 \pm 0.106 & 2.858 \pm 0.156 & 2.015 \pm 0.114 & - & - & - \\ 50 & 0.037 \pm 0.003 & 0.095 \pm 0.006 & 0.077 \pm 0.005 & 0.054 \pm 0.003 & 0.244 \pm 0.012 & 0.139 \pm 0.007 & 1.5 \pm 0.1 & 2.6 \pm 0.2 & 1.8 \pm 0.1 \\ 100 & 0.112 \pm 0.013 & 0.153 \pm 0.017 & 0.388 \pm 0.047 & 0.194 \pm 0.011 & 0.359 \pm 0.019 & 1.113 \pm 0.057 & 1.7 \pm 0.2 & 2.3 \pm 0.3 & 2.9 \pm 0.4 \\ 200 & 0.015 \pm 0.002 & 0.108 \pm 0.008 & 0.022 \pm 0.002 & 0.025 \pm 0.001 & 0.128 \pm 0.007 & 0.034 \pm 0.002 & 1.7 \pm 0.2 & 1.2 \pm 0.1 & 1.5 \pm 0.2\end{array}$

June 2012 P4

$\begin{array}{ccccccccccc}30 & 0.063 \pm 0.003 & 0.044 \pm 0.005 & 0.005 \pm 0.001 & 1.111 \pm 0.029 & 0.285 \pm 0.015 & 0.085 \pm 0.005 & 17.6 \pm 1.1 & 6.4 \pm 0.8 & 15.6 \pm 2.0 \\ 50 & 0.071 \pm 0.006 & 0.032 \pm 0.003 & 0.008 \pm 0.001 & 0.702 \pm 0.036 & 0.208 \pm 0.011 & 0.052 \pm 0.003 & 10.0 \pm 1.0 & 6.5 \pm 0.7 & 6.7 \pm 0.9 \\ 200 & 0.014 \pm 0.002 & 0.026 \pm 0.002 & 0.010 \pm 0.001 & 0.019 \pm 0.001 & 0.058 \pm 0.003 & 0.025 \pm 0.002 & 1.3 \pm 0.2 & 2.2 \pm 0.2 & 2.4 \pm 0.3\end{array}$


June 2012 P12

\begin{tabular}{|c|c|c|c|c|c|c|c|c|c|}
\hline 30 & $0.042 \pm 0.004$ & $0.014 \pm 0.002$ & $0.009 \pm 0.001$ & $0.113 \pm 0.006$ & $0.073 \pm 0.004$ & $0.048 \pm 0.002$ & $2.7 \pm 0.3$ & $5.1 \pm 0.6$ & $5.5 \pm 0.7$ \\
\hline 50 & $0.062 \pm 0.005$ & $0.014 \pm 0.002$ & $0.009 \pm 0.001$ & $0.172 \pm 0.009$ & $0.070 \pm 0.004$ & $0.038 \pm 0.002$ & $2.8 \pm 0.3$ & $4.9 \pm 0.6$ & $4.4 \pm 0.6$ \\
\hline 100 & $0.034 \pm 0.004$ & $0.068 \pm 0.006$ & $0.025 \pm 0.003$ & $0.053 \pm 0.003$ & $0.122 \pm 0.006$ & $0.041 \pm 0.002$ & $1.6 \pm 0.2$ & $1.8 \pm 0.2$ & $1.6 \pm 0.2$ \\
\hline 200 & $0.032 \pm 0.003$ & $0.070 \pm 0.006$ & $0.018 \pm 0.002$ & $0.056 \pm 0.003$ & $0.102 \pm 0.005$ & $0.032 \pm 0.002$ & $1.8 \pm 0.2$ & $1.5 \pm 0.1$ & $1.8 \pm 0.2$ \\
\hline \multicolumn{10}{|c|}{ June 2012 P16 } \\
\hline 30 & $0.131 \pm 0.009$ & $0.014 \pm 0.002$ & $0.014 \pm 0.002$ & $0.398 \pm 0.020$ & $0.084 \pm 0.004$ & $0.054 \pm 0.003$ & $3.0 \pm 0.3$ & $6.1 \pm 0.8$ & $3.9 \pm 0.5$ \\
\hline 50 & $0.127 \pm 0.010$ & $0.031 \pm 0.003$ & $0.022 \pm 0.002$ & $0.230 \pm 0.012$ & $0.091 \pm 0.005$ & $0.050 \pm 0.003$ & $1.8 \pm 0.2$ & $2.9 \pm 0.3$ & $2.3 \pm 0.3$ \\
\hline 100 & $0.035 \pm 0.003$ & $0.036 \pm 0.003$ & $0.019 \pm 0.002$ & $0.076 \pm 0.004$ & $0.081 \pm 0.004$ & $0.026 \pm 0.002$ & $2.2 \pm 0.2$ & $2.2 \pm 0.2$ & $1.4 \pm 0.2$ \\
\hline 200 & $0.035 \pm 0.004$ & $0.055 \pm 0.006$ & $0.022 \pm 0.003$ & $0.050 \pm 0.003$ & $0.075 \pm 0.004$ & $0.034 \pm 0.002$ & $1.4 \pm 0.2$ & $1.4 \pm 0.2$ & $1.5 \pm 0.2$ \\
\hline \multicolumn{10}{|c|}{ June 2012 P20 } \\
\hline 30 & $0.032 \pm 0.004$ & $0.018 \pm 0.002$ & $0.011 \pm 0.001$ & $0.060 \pm 0.003$ & $0.079 \pm 0.004$ & $0.044 \pm 0.002$ & $1.9 \pm 0.2$ & $4.3 \pm 0.5$ & $3.9 \pm 0.5$ \\
\hline 50 & $0.040 \pm 0.004$ & $0.024 \pm 0.003$ & $0.017 \pm 0.002$ & $0.071 \pm 0.003$ & $0.081 \pm 0.004$ & $0.040 \pm 0.002$ & $1.8 \pm 0.2$ & $3.4 \pm 0.4$ & $2.4 \pm 0.3$ \\
\hline 100 & $0.032 \pm 0.003$ & $0.102 \pm 0.009$ & $0.044 \pm 0.004$ & $0.025 \pm 0.001$ & $0.113 \pm 0.006$ & $0.024 \pm 0.001$ & $0.8 \pm 0.1$ & $1.1 \pm 0.1$ & $0.6 \pm 0.1$ \\
\hline 200 & $0.021 \pm 0.003$ & $0.098 \pm 0.009$ & $0.023 \pm 0.003$ & $0.011 \pm 0.000$ & $0.065 \pm 0.003$ & $0.002 \pm 0.000$ & $0.5 \pm 0.1$ & $0.7 \pm 0.1$ & $0.1 \pm 0.0$ \\
\hline \multicolumn{10}{|c|}{ June 2012 P26 } \\
\hline 30 & $0.098 \pm 0.007$ & $0.046 \pm 0.004$ & $0.096 \pm 0.007$ & $0.332 \pm 0.017$ & $0.178 \pm 0.009$ & $0.191 \pm 0.009$ & $3.4 \pm 0.3$ & $3.9 \pm 0.4$ & $2.0 \pm 0.2$ \\
\hline 50 & $0.041 \pm 0.004$ & $0.043 \pm 0.004$ & $0.040 \pm 0.004$ & $0.097 \pm 0.005$ & $0.082 \pm 0.004$ & $0.054 \pm 0.003$ & $2.4 \pm 0.3$ & $1.9 \pm 0.2$ & $1.3 \pm 0.1$ \\
\hline 100 & $0.025 \pm 0.003$ & $0.029 \pm 0.003$ & $0.018 \pm 0.002$ & $0.040 \pm 0.002$ & $0.072 \pm 0.004$ & $0.015 \pm 0.001$ & $1.6 \pm 0.2$ & $2.5 \pm 0.3$ & $0.9 \pm 0.1$ \\
\hline 200 & $0.012 \pm 0.002$ & $0.028 \pm 0.003$ & $0.010 \pm 0.001$ & $0.013 \pm 0.001$ & $0.059 \pm 0.003$ & $0.014 \pm 0.001$ & $1.1 \pm 0.1$ & $2.1 \pm 0.3$ & $1.3 \pm 0.2$ \\
\hline
\end{tabular}


Table S3: Size-fractionated pigment proportion factors (PF) from particles collected by in situ pump and water column PFs from Niskin bottle samples.

\begin{tabular}{ccccccccccccc}
\hline \multicolumn{10}{c}{ Pump } & \multicolumn{1}{c}{ Bottle } \\
\hline \multicolumn{1}{c}{} & \multicolumn{1}{c}{$10-53 \mu \mathrm{m}$} & \multicolumn{1}{c}{$1-10 \mu \mathrm{m}$} \\
Depth (m) & $\mathrm{mPF}$ & $\mathrm{nPF}$ & $\mathrm{pPF}$ & $\mathrm{mPF}$ & $\mathrm{nPF}$ & $\mathrm{pPF}$ & $\mathrm{mPF}$ & $\mathrm{nPF}$ & $\mathrm{pPF}$ & $\mathrm{mPF}$ & $\mathrm{nPF}$ & $\mathrm{pPF}$ \\
\hline Aug. 2010 P12 & & & & & & & & & & & & \\
10 & - & - & - & - & - & - & - & - & - & 0.208 & 0.394 & 0.398 \\
20 & - & - & - & - & - & - & - & - & - & 0.233 & 0.331 & 0.436 \\
30 & - & - & - & - & - & - & - & - & - & 0.211 & 0.533 & 0.257 \\
75 & - & - & - & - & - & - & - & - & - & 0.150 & 0.617 & 0.233
\end{tabular}

Aug. 2010 P16

\begin{tabular}{|c|c|c|c|c|c|c|c|c|c|c|c|}
\hline 10 & - & - & - & 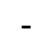 & - & - & - & - & 0.197 & 0.574 & 0.230 \\
\hline 30 & - & - & - & - & - & - & - & - & 0.143 & 0.615 & 0.242 \\
\hline 75 & - & - & - & - & - & - & - & - & 0.115 & 0.648 & 0.238 \\
\hline
\end{tabular}

Aug. 2010 P20

\begin{tabular}{|c|c|c|c|c|c|c|c|c|c|c|c|}
\hline 10 & - & - & - & - & - & - & - & - & 0.170 & 0.660 & 0.170 \\
\hline 30 & - & - & - & - & - & - & 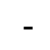 & - & 0.313 & 0.511 & 0.176 \\
\hline 75 & - & - & - & - & - & - & - & - & 0.113 & 0.616 & 0.272 \\
\hline
\end{tabular}

Feb. 2011 P12

$\begin{array}{lllllllllllll}1 & - & - & - & - & - & - & - & - & - & 0.153 & 0.441 & 0.405 \\ 43 & - & - & - & - & - & - & - & - & - & 0.164 & 0.444 & 0.392 \\ 55 & - & - & - & - & - & - & - & - & - & 0.167 & 0.443 & 0.390 \\ 65 & - & - & - & - & - & - & - & - & - & 0.157 & 0.418 & 0.425\end{array}$

Feb. 2011 P16

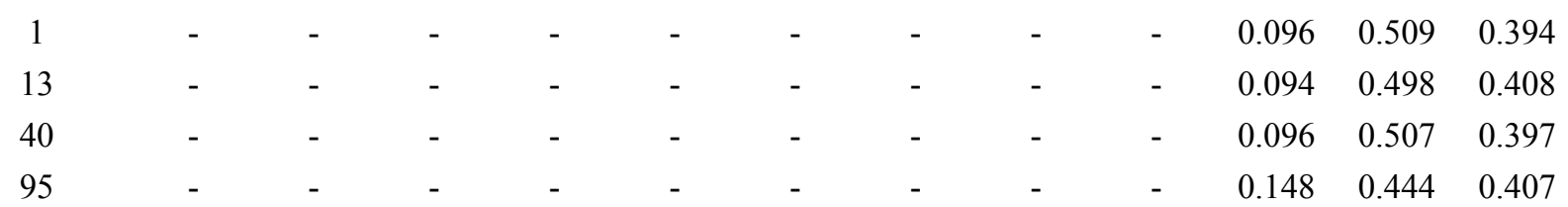

Feb. 2011 P20

\begin{tabular}{|c|c|c|c|c|c|c|c|c|c|c|c|c|}
\hline 1 & - & - & - & - & - & - & - & - & - & 0.130 & 0.541 & 0.329 \\
\hline 11 & - & - & - & - & - & - & - & - & - & 0.127 & 0.532 & 0.342 \\
\hline 38 & - & - & - & - & - & - & - & - & - & 0.126 & 0.551 & 0.323 \\
\hline 77 & - & - & - & - & - & - & - & - & - & 0.112 & 0.532 & 0.356 \\
\hline
\end{tabular}

June $2011 \mathrm{P} 4$

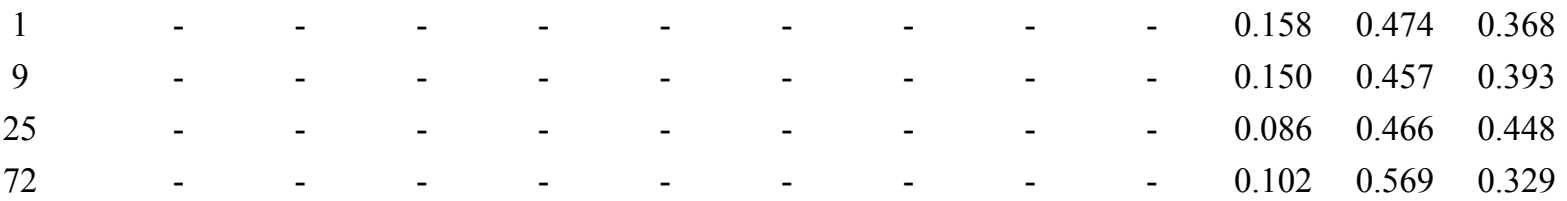


June 2011 P12

$\begin{array}{lllllllllllll}5 & - & - & - & - & - & - & - & - & - & 0.138 & 0.460 & 0.402 \\ 15 & - & - & - & - & - & - & - & - & - & 0.142 & 0.486 & 0.372 \\ 40 & - & - & - & - & - & - & - & - & - & 0.204 & 0.675 & 0.121 \\ 90 & - & - & - & - & - & - & - & - & - & 0.257 & 0.537 & 0.206\end{array}$

June 2011 P16

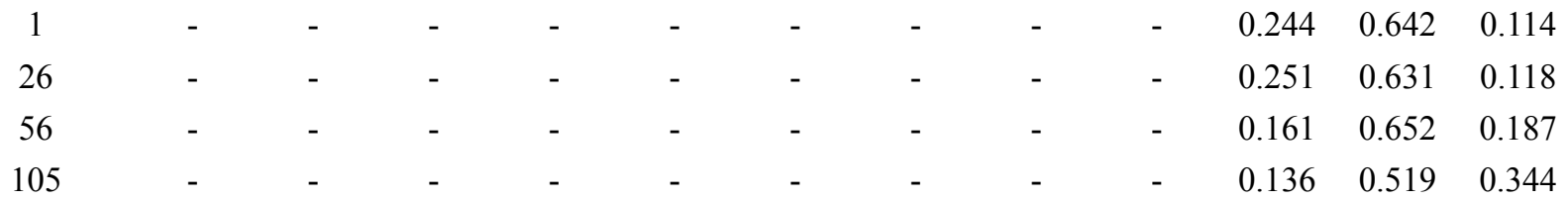

June 2011 P20

\begin{tabular}{|c|c|c|c|c|c|c|c|c|c|c|c|c|}
\hline 1 & - & - & - & - & - & - & - & - & - & 0.231 & 0.615 & 0.154 \\
\hline 15 & - & - & - & - & - & - & - & - & - & 0.289 & 0.560 & 0.151 \\
\hline 34 & - & - & - & - & - & - & - & - & - & 0.274 & 0.605 & 0.121 \\
\hline 70 & - & - & - & - & - & - & - & - & - & 0.180 & 0.429 & 0.392 \\
\hline
\end{tabular}

June 2011 P26

$\begin{array}{ccccccccccccc}1 & - & - & - & - & - & - & - & - & - & 0.257 & 0.493 & 0.250 \\ 5 & - & - & - & - & - & - & - & - & - & 0.246 & 0.490 & 0.264 \\ 9 & - & - & - & - & - & - & - & - & - & 0.227 & 0.449 & 0.324 \\ 10 & - & - & - & - & - & - & - & - & - & 0.281 & 0.506 & 0.213 \\ 14 & - & - & - & - & - & - & - & - & - & 0.276 & 0.509 & 0.215 \\ 16 & - & - & - & - & - & - & - & - & - & 0.219 & 0.470 & 0.310 \\ 24 & - & - & - & - & - & - & - & - & - & 0.261 & 0.492 & 0.247 \\ 30 & 0.545 & 0.351 & 0.104 & 0.487 & 0.442 & 0.071 & 0.421 & 0.498 & 0.081 & - & - & - \\ 49 & - & - & - & - & - & - & - & - & - & 0.295 & 0.539 & 0.166 \\ 50 & 0.630 & 0.288 & 0.083 & 0.606 & 0.326 & 0.067 & 0.496 & 0.431 & 0.073 & - & - & - \\ 52 & - & - & - & - & - & - & - & - & - & 0.223 & 0.580 & 0.198 \\ 83 & - & - & - & - & - & - & - & - & - & 0.264 & 0.431 & 0.305 \\ 85 & - & - & - & - & - & - & - & - & - & 0.241 & 0.580 & 0.180 \\ 100 & 0.809 & 0.119 & 0.072 & 0.729 & 0.198 & 0.073 & 0.712 & 0.225 & 0.063 & & & \\ 150 & 0.505 & 0.495 & 0.000 & 0.532 & 0.327 & 0.142 & 0.526 & 0.474 & 0.000 & & & \\ 200 & 0.585 & 0.226 & 0.189 & 0.590 & 0.161 & 0.249 & 0.451 & 0.332 & 0.217 & & & \end{array}$

Feb. 2012 P4

$\begin{array}{ccccccccccccc}1 & - & - & - & - & - & - & - & - & - & 0.267 & 0.482 & 0.252 \\ 8 & - & - & - & - & - & - & - & - & - & 0.270 & 0.483 & 0.247 \\ 14 & - & - & - & - & - & - & - & - & - & 0.280 & 0.458 & 0.263 \\ 22 & - & - & - & - & - & - & - & - & - & 0.252 & 0.458 & 0.290 \\ 30 & 0.667 & 0.317 & 0.016 & 0.660 & 0.278 & 0.062 & 0.543 & 0.376 & 0.080 & - & - & - \\ 35 & - & - & - & - & - & - & - & - & - & 0.227 & 0.479 & 0.294 \\ 50 & 0.666 & 0.334 & 0.000 & 0.638 & 0.269 & 0.094 & 0.527 & 0.397 & 0.076 & 0.248 & 0.461 & 0.291 \\ 100 & 0.615 & 0.385 & 0.000 & 0.529 & 0.354 & 0.117 & 0.584 & 0.332 & 0.085 & - & - & -\end{array}$


Feb. 2012 P12

$\begin{array}{ccccccccccccc}1 & - & - & - & - & - & - & - & - & - & 0.149 & 0.656 & 0.195 \\ 8 & - & - & - & - & - & - & - & - & - & 0.151 & 0.650 & 0.199 \\ 12 & - & - & - & - & - & - & - & - & - & 0.136 & 0.645 & 0.219 \\ 26 & - & - & - & - & - & - & - & - & - & 0.126 & 0.662 & 0.212 \\ 30 & 0.633 & 0.291 & 0.076 & 0.431 & 0.481 & 0.088 & 0.279 & 0.568 & 0.153 & - & - & - \\ 41 & - & - & - & - & - & - & - & - & - & 0.124 & 0.659 & 0.216 \\ 50 & 0.514 & 0.396 & 0.089 & 0.380 & 0.492 & 0.128 & 0.234 & 0.517 & 0.249 & - & - & - \\ 55 & - & - & - & - & - & - & - & - & - & 0.140 & 0.683 & 0.177 \\ 95 & - & - & - & - & - & - & - & - & - & 0.192 & 0.515 & 0.293 \\ 100 & 0.509 & 0.491 & 0.000 & 0.330 & 0.501 & 0.169 & 0.435 & 0.565 & 0.000 & - & - & - \\ 200 & 0.283 & 0.717 & 0.000 & 0.480 & 0.393 & 0.127 & 0.251 & 0.749 & 0.000 & - & - & -\end{array}$

Feb. 2012 P26

$\begin{array}{ccccccccccccc}1 & - & - & - & - & - & - & - & - & - & 0.296 & 0.540 & 0.164 \\ 10 & - & - & - & - & - & - & - & - & - & 0.296 & 0.564 & 0.140 \\ 15 & - & - & - & - & - & - & - & - & - & 0.286 & 0.574 & 0.140 \\ 25 & - & - & - & - & - & - & - & - & - & 0.494 & 0.411 & 0.096 \\ 30 & 0.680 & 0.265 & 0.055 & 0.799 & 0.201 & 0.000 & 0.699 & 0.301 & 0.000 & - & - & - \\ 36 & - & - & - & - & - & - & - & - & - & 0.295 & 0.586 & 0.119 \\ 47 & - & - & - & - & - & - & - & - & - & 0.293 & 0.534 & 0.173 \\ 50 & 0.778 & 0.172 & 0.050 & 0.672 & 0.229 & 0.099 & 0.654 & 0.258 & 0.088 & - & - & - \\ 75 & - & - & - & - & - & - & - & - & - & 0.281 & 0.556 & 0.163 \\ 100 & 0.666 & 0.197 & 0.138 & 0.596 & 0.253 & 0.151 & 0.590 & 0.268 & 0.142 & - & - & - \\ 200 & 0.734 & 0.266 & 0.000 & 0.502 & 0.237 & 0.261 & 0.668 & 0.332 & 0.000 & - & - & -\end{array}$

June 2012 P4

$\begin{array}{ccccccccccccc}2 & - & - & - & - & - & - & - & - & - & 0.747 & 0.253 & 0.000 \\ 18 & - & - & - & - & - & - & - & - & - & 0.791 & 0.209 & 0.000 \\ 30 & 0.720 & 0.158 & 0.122 & 0.902 & 0.072 & 0.026 & 0.883 & 0.100 & 0.017 & - & - & - \\ 31 & - & - & - & - & - & - & - & - & - & 0.862 & 0.138 & 0.000 \\ 50 & - & - & - & - & - & - & 0.884 & 0.069 & 0.048 & - & - & - \\ 103 & - & - & - & - & - & - & - & - & - & 1.000 & 0.000 & 0.000 \\ 200 & 0.856 & 0.112 & 0.032 & - & - & - & 0.851 & 0.149 & 0.000 & - & - & -\end{array}$

June 2012 P12

$\begin{array}{ccccccccccccc}6 & - & - & - & - & - & - & - & - & - & 0.202 & 0.798 & 0.000 \\ 30 & 0.555 & 0.412 & 0.033 & 0.576 & 0.386 & 0.038 & - & - & - & - & - & - \\ 50 & 0.552 & 0.388 & 0.060 & 0.595 & 0.359 & 0.046 & 0.381 & 0.490 & 0.129 & - & - & - \\ 100 & 0.738 & 0.213 & 0.049 & 0.541 & 0.369 & 0.090 & 0.416 & 0.517 & 0.067 & - & - & - \\ 111 & - & - & - & - & - & - & - & - & - & 0.142 & 0.858 & 0.000 \\ 164 & - & - & - & - & - & - & - & - & - & 1.000 & 0.000 & 0.000\end{array}$

$\begin{array}{llllllllll}200 & 0.298 & 0.542 & 0.161 & 0.309 & 0.589 & 0.103 & 0.247 & 0.753 & 0.000\end{array}$ 


$\begin{array}{ccccccccccccc}\text { June } 2012 \text { P16 } & & & & & & & & & & & & \\ 5 & - & - & - & - & - & - & - & - & - & 0.176 & 0.724 & 0.100 \\ 16 & - & - & - & - & - & - & - & - & - & 0.189 & 0.597 & 0.214 \\ 30 & - & - & - & - & - & - & 0.599 & 0.339 & 0.063 & - & - & - \\ 50 & 0.496 & 0.372 & 0.132 & 0.539 & 0.420 & 0.041 & - & - & - & - & - & - \\ 66 & - & - & - & - & - & - & - & - & - & 0.383 & 0.617 & 0.000 \\ 100 & 0.877 & 0.123 & 0.000 & 0.794 & 0.167 & 0.038 & 0.646 & 0.284 & 0.071 & - & - & - \\ 200 & 0.480 & 0.520 & 0.000 & - & - & - & 0.321 & 0.679 & 0.000 & - & - & -\end{array}$

June 2012 P20

$\begin{array}{ccccccccccccc}30 & 0.434 & 0.355 & 0.211 & 0.634 & 0.258 & 0.108 & 0.388 & 0.429 & 0.182 & - & - & - \\ 50 & - & - & - & - & - & - & - & - & - & - & - \\ 100 & - & - & - & 0.687 & 0.222 & 0.091 & 0.410 & 0.507 & 0.084 & - & - \\ 200 & 0.437 & 0.563 & 0.000 & 0.334 & 0.506 & 0.160 & 0.343 & 0.657 & 0.000 & - & - & -\end{array}$

June 2012 P26

\begin{tabular}{ccccccccccccc}
30 & 0.650 & 0.307 & 0.043 & 0.791 & 0.209 & 0.000 & 0.516 & 0.452 & 0.032 & - & - & - \\
50 & 0.586 & 0.358 & 0.056 & 0.628 & 0.306 & 0.066 & 0.490 & 0.468 & 0.043 & - & - & - \\
100 & - & - & - & 0.693 & 0.217 & 0.089 & 0.481 & 0.426 & 0.093 & - & - & - \\
200 & 0.533 & 0.467 & 0.000 & - & - & - & - & - & - & - & - & - \\
\hline
\end{tabular}


Table S4: Chl $a$ and indicator pigment concentrations determined by HPLC from in situ pump samples.

\begin{tabular}{|c|c|c|c|c|c|c|c|c|c|}
\hline $\begin{array}{l}\text { Depth } \\
\text { (m) }\end{array}$ & Size-fraction & $\begin{array}{c}\text { Chl } a \\
\left(\text { ng L L }^{-1}\right) \\
\end{array}$ & $\begin{array}{l}\text { FUCO } \\
\left(\operatorname{ng~L}^{-1}\right) \\
\end{array}$ & $\begin{array}{c}\text { PER } \\
\left(\mathrm{ng} \mathrm{L}^{-1}\right) \\
\end{array}$ & $\begin{array}{c}\text { HEX } \\
\left(\operatorname{ng~L}^{-1}\right) \\
\end{array}$ & $\begin{array}{c}\text { BUT } \\
\left(\operatorname{ng~L}^{-1}\right) \\
\end{array}$ & $\begin{array}{l}\text { ALLO } \\
\left(\mathrm{ng} \mathrm{L}^{-1}\right) \\
\end{array}$ & $\begin{array}{c}\text { Chl } b \\
\left(\operatorname{ng~L}^{-1}\right) \\
\end{array}$ & $\begin{array}{c}\begin{array}{c}\text { ZEA } \\
\left(\mathrm{ng} \mathrm{L}^{-1}\right)\end{array} \\
\end{array}$ \\
\hline \multicolumn{10}{|c|}{ June 2011 P26 } \\
\hline 30 & $>53 \mu \mathrm{m}$ & 511.12 & 229.16 & 7.54 & 119.35 & 27.87 & 4.93 & 33.69 & 11.48 \\
\hline 30 & $10-53 \mu \mathrm{m}$ & 673.72 & 264.61 & 9.77 & 188.55 & 56.52 & 3.78 & 25.35 & 14.55 \\
\hline 30 & $1-10 \mu \mathrm{m}$ & 625.27 & 227.05 & 7.24 & 212.78 & 60.45 & 3.92 & 26.18 & 19.07 \\
\hline 50 & $>53 \mu \mathrm{m}$ & 82.39 & 55.73 & 12.81 & 24.99 & 6.35 & - & 5.95 & 3.03 \\
\hline 50 & $10-53 \mu \mathrm{m}$ & 121.63 & 72.34 & 21.95 & 40.85 & 9.91 & - & 6.08 & 4.41 \\
\hline 50 & $1-10 \mu \mathrm{m}$ & 47.43 & 24.51 & 9.17 & 24.14 & 5.13 & - & 3.17 & 1.75 \\
\hline 100 & $>53 \mu \mathrm{m}$ & 33.61 & 20.82 & 1.60 & 2.61 & 0.01 & 0.68 & 1.23 & 0.77 \\
\hline 100 & $10-53 \mu \mathrm{m}$ & 139.07 & 73.64 & 7.63 & 7.69 & 12.98 & 1.36 & 6.95 & 1.20 \\
\hline 100 & $1-10 \mu \mathrm{m}$ & 11.67 & 6.35 & 1.10 & 1.15 & 1.21 & 0.003 & 0.52 & 0.14 \\
\hline 150 & $>53 \mu \mathrm{m}$ & 5.61 & 1.94 & 1.91 & 3.36 & 0.41 & - & - & - \\
\hline 150 & $10-53 \mu \mathrm{m}$ & 18.50 & 7.01 & 4.24 & 5.79 & 1.12 & - & 2.60 & 0.40 \\
\hline 150 & $1-10 \mu \mathrm{m}$ & 3.32 & 1.11 & 0.96 & 1.56 & 0.31 & - & - & - \\
\hline 200 & $>53 \mu \mathrm{m}$ & 6.21 & 3.00 & 1.31 & 1.17 & 0.003 & 0.50 & 0.77 & 0.63 \\
\hline 200 & $10-53 \mu \mathrm{m}$ & 15.53 & 5.93 & 2.52 & 1.46 & 0.01 & 0.84 & 2.86 & 0.70 \\
\hline 200 & $1-10 \mu \mathrm{m}$ & 3.50 & 1.31 & 0.52 & 0.54 & 0.65 & 0.16 & 0.67 & 0.21 \\
\hline \multicolumn{10}{|c|}{ Feb. 2012 P4 } \\
\hline 30 & $>53 \mu \mathrm{m}$ & 40.59 & 20.44 & - & 7.63 & 1.19 & 0.89 & - & 0.49 \\
\hline 30 & $10-53 \mu \mathrm{m}$ & 545.35 & 268.88 & 76.29 & 112.12 & 21.34 & 11.74 & 32.34 & - \\
\hline 30 & $1-10 \mu \mathrm{m}$ & 432.97 & 204.00 & - & 111.84 & 17.21 & 12.35 & 24.57 & 5.60 \\
\hline 50 & $>53 \mu \mathrm{m}$ & 17.62 & 10.00 & - & 4.35 & 0.66 & - & - & - \\
\hline 50 & $10-53 \mu \mathrm{m}$ & 203.71 & 102.04 & 29.95 & 43.46 & 7.72 & 4.39 & 9.15 & 10.24 \\
\hline 50 & $1-10 \mu \mathrm{m}$ & 220.87 & 100.64 & - & 60.78 & 7.97 & 7.06 & 11.86 & 2.75 \\
\hline 100 & $>53 \mu \mathrm{m}$ & 5.28 & 2.85 & - & 1.51 & 0.27 & - & - & - \\
\hline 100 & $10-53 \mu \mathrm{m}$ & 50.51 & 26.90 & 6.81 & 18.45 & 3.15 & 0.99 & 6.68 & 0.78 \\
\hline 100 & $1-10 \mu \mathrm{m}$ & 39.13 & 20.82 & 2.66 & 10.92 & 1.76 & 0.67 & 2.65 & 0.76 \\
\hline 150 & $>53 \mu \mathrm{m}$ & 3.26 & 1.86 & 0.67 & 1.23 & 0.47 & - & - & 1.48 \\
\hline 150 & $10-53 \mu \mathrm{m}$ & 19.19 & 7.44 & 2.90 & 7.78 & 0.97 & 0.67 & 1.78 & - \\
\hline 150 & $1-10 \mu \mathrm{m}$ & 4.77 & 2.41 & 0.00 & 1.68 & - & - & - & - \\
\hline \multicolumn{10}{|c|}{ Feb. 2012 P12 } \\
\hline 30 & $>53 \mu \mathrm{m}$ & 20.81 & 12.90 & 1.96 & 4.66 & 2.18 & - & 1.78 & - \\
\hline 30 & $10-53 \mu \mathrm{m}$ & 297.72 & 105.62 & 40.44 & 126.95 & 32.07 & 4.14 & 26.73 & 3.25 \\
\hline 30 & $1-10 \mu \mathrm{m}$ & 86.74 & 18.82 & 10.23 & 44.53 & 12.84 & 1.74 & 14.36 & 1.55 \\
\hline 50 & $>53 \mu \mathrm{m}$ & 103.21 & 62.46 & 16.84 & 41.04 & 9.41 & 10.66 & 11.97 & 1.78 \\
\hline 50 & $10-53 \mu \mathrm{m}$ & 253.18 & 71.78 & 27.49 & 95.03 & 28.12 & 5.27 & 30.50 & 3.06 \\
\hline 50 & $1-10 \mu \mathrm{m}$ & 159.39 & 26.82 & 13.44 & 63.47 & 23.15 & 2.49 & 40.10 & 2.78 \\
\hline 100 & $>53 \mu \mathrm{m}$ & 2.24 & 0.97 & - & 0.70 & 0.23 & - & - & - \\
\hline 100 & $10-53 \mu \mathrm{m}$ & 3.24 & 0.72 & 0.53 & 1.56 & 0.33 & - & 0.64 & - \\
\hline 100 & $1-10 \mu \mathrm{m}$ & 2.67 & 1.09 & - & 1.04 & 0.36 & - & - & - \\
\hline 200 & $>53 \mu \mathrm{m}$ & 1.72 & 0.39 & - & 0.69 & 0.28 & - & - & - \\
\hline 200 & $10-53 \mu \mathrm{m}$ & 22.18 & 6.25 & 3.58 & 4.64 & 1.77 & 1.65 & 2.60 & - \\
\hline 200 & $1-10 \mu \mathrm{m}$ & 4.75 & 0.93 & - & 2.08 & 0.68 & - & - & - \\
\hline \multicolumn{10}{|c|}{ Feb. 2012 P26 } \\
\hline 30 & $>53 \mu \mathrm{m}$ & 1355.87 & 597.74 & 332.35 & 271.35 & 91.56 & - & 74.59 & - \\
\hline 30 & $10-53 \mu \mathrm{m}$ & 437.92 & 231.80 & 86.08 & 50.77 & 29.04 & - & - & - \\
\hline 30 & $1-10 \mu \mathrm{m}$ & 188.72 & 73.00 & 63.13 & 34.57 & 24.05 & - & - & - \\
\hline 50 & $>53 \mu \mathrm{m}$ & 76.53 & 64.69 & 10.68 & 9.77 & 6.31 & 0.59 & 3.56 & 1.23 \\
\hline 50 & $10-53 \mu \mathrm{m}$ & 452.25 & 227.96 & 92.37 & 75.94 & 26.42 & 7.00 & 40.92 & 6.31 \\
\hline 50 & $1-10 \mu \mathrm{m}$ & 434.35 & 178.31 & 131.28 & 85.77 & 28.27 & 8.09 & 34.14 & 7.47 \\
\hline 100 & $>53 \mu \mathrm{m}$ & 110.25 & 72.78 & 10.23 & 13.40 & 11.11 & - & 13.19 & 3.99 \\
\hline
\end{tabular}




$\begin{array}{cccccccccc}100 & 10-53 \mu \mathrm{m} & 115.33 & 57.53 & 12.36 & 20.41 & 9.22 & - & 17.65 & - \\ 100 & 1-10 \mu \mathrm{m} & 125.06 & 53.84 & 36.95 & 27.93 & 9.40 & 3.95 & 19.38 & 2.45 \\ 200 & >53 \mu \mathrm{m} & 1.49 & 0.56 & 0.92 & 0.35 & 0.19 & - & - & - \\ 200 & 10-53 \mu \mathrm{m} & 11.51 & 3.31 & 4.43 & 2.23 & 0.97 & 0.45 & 4.02 & - \\ 200 & 1-10 \mu \mathrm{m} & 1.95 & 0.51 & 0.76 & 0.41 & 0.22 & - & - & -\end{array}$

June 2012 P4

$\begin{array}{cccccccccc}30 & >53 \mu \mathrm{m} & 230.87 & 223.78 & 19.14 & 42.55 & - & 10.71 & 13.92 & 27.17 \\ 30 & 10-53 \mu \mathrm{m} & 352.30 & 236.17 & - & 18.89 & - & - & - & 6.85 \\ 30 & 1-10 \mu \mathrm{m} & 117.89 & 73.57 & 4.75 & 7.08 & - & 1.74 & - & 1.53 \\ 50 & >53 \mu \mathrm{m} & 557.66 & 423.75 & 29.72 & 22.22 & - & - & - & 14.00 \\ 50 & 10-53 \mu \mathrm{m} & 479.75 & 329.71 & 19.32 & 13.46 & - & - & - & - \\ 50 & 1-10 \mu \mathrm{m} & 61.77 & 48.71 & 5.09 & 4.19 & - & - & 1.49 & 1.41 \\ 200 & >53 \mu \mathrm{m} & 10.41 & 8.90 & 0.46 & 1.22 & - & - & - & 0.35 \\ 200 & 10-53 \mu \mathrm{m} & 20.83 & 18.84 & - & 3.20 & - & - & - & 0.94 \\ 200 & 1-10 \mu \mathrm{m} & 1.96 & 2.69 & - & 0.47 & - & - & - & -\end{array}$

June 2012 P12

$\begin{array}{cccccccccc}30 & >53 \mu \mathrm{m} & 42.53 & 23.34 & - & 13.35 & 3.96 & - & 1.37 & - \\ 30 & 10-53 \mu \mathrm{m} & 33.69 & 16.84 & - & 10.02 & 1.26 & - & 1.11 & - \\ 30 & 1-10 \mu \mathrm{m} & 10.89 & 3.79 & - & 4.98 & 0.55 & - & 0.98 & - \\ 50 & >53 \mu \mathrm{m} & 60.19 & 31.10 & - & 15.81 & 6.03 & - & 2.58 & 0.80 \\ 50 & 10-53 \mu \mathrm{m} & 34.02 & 23.14 & - & 11.89 & 2.07 & - & 1.80 & - \\ 50 & 1-10 \mu \mathrm{m} & 4.65 & 2.39 & - & 2.74 & 0.33 & - & 0.81 & - \\ 100 & >53 \mu \mathrm{m} & 15.59 & 11.04 & 4.48 & 3.66 & 0.82 & - & 1.04 & - \\ 100 & 10-53 \mu \mathrm{m} & 38.28 & 20.46 & 6.75 & 14.10 & 4.45 & - & 4.53 & - \\ 100 & 1-10 \mu \mathrm{m} & 2.28 & 7.55 & 1.23 & 9.12 & 1.77 & - & 1.42 & - \\ 200 & >53 \mu \mathrm{m} & 1.25 & 1.14 & - & 1.83 & 0.25 & - & 0.62 & - \\ 200 & 10-53 \mu \mathrm{m} & 1.61 & 1.43 & - & 2.72 & - & - & 0.47 & - \\ 200 & 1-10 \mu \mathrm{m} & 0.37 & 0.20 & - & 0.61 & - & - & - & -\end{array}$

June 2012 P16

$\begin{array}{cccccccccc}30 & >53 \mu \mathrm{m} & 26.25 & 13.87 & - & 3.85 & 0.54 & - & 2.87 & \\ 30 & 10-53 \mu \mathrm{m} & 9.10 & 5.90 & - & 1.83 & 0.31 & - & - & 0.23 \\ 30 & 1-10 \mu \mathrm{m} & 6.95 & 4.00 & 0.57 & 2.25 & 0.34 & - & 0.25 & 0.78 \\ 50 & >53 \mu \mathrm{m} & 18.31 & 10.14 & 1.45 & 7.60 & 0.58 & 0.52 & 2.31 & - \\ 50 & 10-53 \mu \mathrm{m} & 12.96 & 7.26 & 1.31 & 5.84 & 0.83 & - & 0.65 & - \\ 50 & 1-10 \mu \mathrm{m} & 7.04 & 3.79 & 0.62 & 5.25 & 0.30 & - & 0.40 & - \\ 100 & >53 \mu \mathrm{m} & 8.68 & 8.89 & 1.25 & 1.22 & 0.20 & - & - & - \\ 100 & 10-53 \mu \mathrm{m} & 21.76 & 16.93 & 2.10 & 2.17 & 1.84 & - & 0.92 & - \\ 100 & 1-10 \mu \mathrm{m} & 3.21 & 3.73 & 0.71 & 1.62 & 0.33 & - & 0.48 & - \\ 200 & >53 \mu \mathrm{m} & 0.33 & 0.29 & - & 0.32 & - & - & - & - \\ 200 & 10-53 \mu \mathrm{m} & 1.31 & 1.24 & & 0.42 & - & - & - & - \\ 200 & 1-10 \mu \mathrm{m} & - & 0.05 & - & 0.10 & - & - & - & \\ & & & & & & & & & \\ \text { June } 2012 \mathrm{P} 20 & >53 \mu \mathrm{m} & 2.00 & 1.48 & - & 1.03 & 0.18 & - & 0.25 & 0.47 \\ 30 & 10-53 \mu \mathrm{m} & 5.04 & 3.14 & 0.36 & 1.05 & 0.38 & - & 0.34 & 0.26 \\ 30 & 1-10 \mu \mathrm{m} & 2.03 & 0.90 & 0.20 & 0.92 & 0.30 & - & 0.27 & 0.25 \\ 30 & >53 \mu \mathrm{m} & 2.41 & 1.89 & 0.35 & - & 0.31 & - & 0.78 & 0.31 \\ 50 & 10-53 \mu \mathrm{m} & 9.69 & 7.37 & 1.60 & - & 0.46 & - & 1.32 & - \\ 50 & 1-10 \mu \mathrm{m} & 3.22 & 2.24 & 1.34 & - & 0.23 & - & 0.41 & - \\ 50 & >53 \mu \mathrm{m} & 0.61 & 0.61 & 0.15 & - & 0.08 & - & 0.18 & 0.04 \\ 100 & 10-53 \mu \mathrm{m} & 5.46 & 3.81 & 0.71 & 0.85 & 0.61 & - & 0.60 & - \\ 100 & 1-10 \mu \mathrm{m} & 0.65 & 0.58 & 0.08 & 0.65 & 0.17 & - & 0.14 & - \\ 100 & >53 \mu \mathrm{m} & 0.16 & 0.14 & - & 0.18 & - & - & 0.00 & - \\ 200 & & & & & & & \end{array}$




\begin{tabular}{|c|c|c|c|c|c|c|c|c|c|}
\hline 200 & $10-53 \mu \mathrm{m}$ & 0.49 & 0.38 & - & 0.58 & - & - & 0.18 & - \\
\hline 200 & $1-10 \mu \mathrm{m}$ & - & 0.06 & - & 0.12 & - & - & - & - \\
\hline \multicolumn{10}{|c|}{ June 2012 P26 } \\
\hline 30 & $>53 \mu \mathrm{m}$ & 72.84 & 41.00 & - & 16.83 & 2.54 & - & 2.72 & - \\
\hline 30 & $10-53 \mu \mathrm{m}$ & 62.61 & 39.83 & 1.45 & 8.21 & 2.71 & - & - & - \\
\hline 30 & $1-10 \mu \mathrm{m}$ & 63.80 & 32.56 & 1.17 & 21.47 & 8.08 & - & 2.08 & - \\
\hline 50 & $>53 \mu \mathrm{m}$ & 10.90 & 7.21 & 0.76 & 3.89 & 0.98 & - & 0.76 & - \\
\hline 50 & $10-53 \mu \mathrm{m}$ & 26.13 & 15.79 & 1.84 & 6.98 & 1.62 & - & 1.86 & - \\
\hline 50 & $1-10 \mu \mathrm{m}$ & 16.68 & 6.53 & 4.24 & 8.60 & 1.70 & - & 0.94 & - \\
\hline 100 & $>53 \mu \mathrm{m}$ & 3.93 & 4.67 & - & - & 0.45 & - & 0.39 & - \\
\hline 100 & $10-53 \mu \mathrm{m}$ & 7.03 & 5.06 & 0.69 & 1.24 & 0.56 & - & 0.61 & 0.13 \\
\hline 100 & $1-10 \mu \mathrm{m}$ & 2.56 & 2.49 & 0.29 & 1.98 & 0.48 & - & 0.54 & - \\
\hline 200 & $>53 \mu \mathrm{m}$ & 0.20 & 0.22 & - & 0.14 & 0.05 & - & - & - \\
\hline 200 & $10-53 \mu \mathrm{m}$ & 0.45 & 0.48 & - & - & - & - & - & - \\
\hline 200 & $1-10 \mu \mathrm{m}$ & 0.07 & 0.11 & - & - & 0.05 & - & - & - \\
\hline
\end{tabular}


Table S5: Total ${ }^{234} \mathrm{Th}$ and ${ }^{238} \mathrm{U}$ activities, ${ }^{234} \mathrm{Th} /{ }^{238} \mathrm{U}$ activity ratios, and ${ }^{234} \mathrm{Th}$ fluxes.

\begin{tabular}{|c|c|c|c|c|c|}
\hline $\begin{array}{l}\text { Depth } \\
\text { (m) }\end{array}$ & 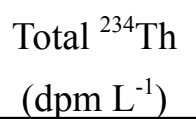 & $\begin{array}{c}{ }^{238} \mathrm{U} \\
\left(\mathrm{dpm} \mathrm{L} \mathrm{L}^{-1}\right)\end{array}$ & ${ }^{234} \mathrm{Th} /{ }^{238} \mathrm{U}$ AR & 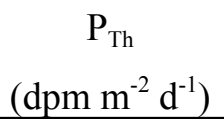 & $\begin{array}{c}\mathrm{F}_{\mathrm{Th}} \\
\left(\mathrm{dpm} \mathrm{m}^{-2} \mathrm{~d}^{-1}\right)\end{array}$ \\
\hline
\end{tabular}

Aug. 2010 P4

$\begin{array}{cccccc}5 & 0.73 \pm 0.06 & 2.27 & 0.32 & - & - \\ 10 & 1.08 \pm 0.09 & 2.27 & 0.47 & - & - \\ 20 & - & 2.28 & - & - & - \\ 21 & 1.33 \pm 0.10 & 2.28 & 0.58 & - & - \\ 30 & 1.81 \pm 0.12 & 2.29 & 0.79 & 794 \pm 97 & - \\ 50 & 2.26 \pm 0.15 & 2.30 & 0.98 & 944 \pm 163 & - \\ 75 & 2.25 \pm 0.17 & 2.32 & 0.97 & - & - \\ 100 & 2.69 \pm 0.17 & 2.36 & 1.14 & 894 \pm 393 & - \\ 150 & 2.06 \pm 0.13 & 2.39 & 0.86 & 901 \pm 611 & - \\ 200 & - & 2.40 & - & 1466 \pm 830 & - \\ 300 & 2.43 \pm 0.16 & 2.41 & 1.01 & - & - \\ 500 & 2.36 \pm 0.16 & 2.42 & 0.98 & - & -\end{array}$

Aug. 2010 P12

$\begin{array}{cccccc}5 & 1.10 \pm 0.08 & 2.24 & 0.49 & - & - \\ 10 & 1.12 \pm 0.08 & 2.24 & 0.50 & - & - \\ 20 & 0.78 \pm 0.06 & 2.24 & 0.35 & - & - \\ 27 & 1.48 \pm 0.11 & 2.28 & 0.65 & - & - \\ 30 & 1.49 \pm 0.11 & 2.30 & 0.65 & 786 \pm 71 & - \\ 50 & 1.80 \pm 0.12 & 2.30 & 0.78 & 1163 \pm 132 & - \\ 75 & 1.84 \pm 0.13 & 2.30 & 0.80 & - & - \\ 100 & 2.06 \pm 0.13 & 2.32 & 0.89 & 1774 \pm 310 & - \\ 150 & 2.35 \pm 0.15 & 2.36 & 0.99 & 1972 \pm 513 & - \\ 200 & 2.52 \pm 0.17 & 2.39 & 1.05 & 1891 \pm 744 & - \\ 300 & 2.19 \pm 0.15 & 2.40 & 0.91 & - & - \\ 500 & 2.14 \pm 0.14 & 2.41 & 0.89 & & -\end{array}$

\section{Aug. 2010 P16}

$\begin{array}{cccc}5 & 1.49 \pm 0.10 & 2.30 & 0.65 \\ 10 & 1.84 \pm 0.14 & 2.30 & 0.80 \\ 20 & 1.54 \pm 0.11 & 2.30 & 0.67 \\ 30 & 0.96 \pm 0.07 & 2.30 & 0.42 \\ 40 & 1.66 \pm 0.11 & 2.31 & 0.72 \\ 50 & 1.87 \pm 0.12 & 2.31 & 0.81 \\ 75 & 2.21 \pm 0.15 & 2.32 & 0.95 \\ 100 & 2.23 \pm 0.15 & 2.33 & 0.96 \\ 150 & 2.33 \pm 0.16 & 2.39 & 0.97 \\ 200 & 2.06 \pm 0.13 & 2.39 & 0.86 \\ 300 & 2.38 \pm 0.16 & 2.40 & 0.99 \\ 500 & 2.50 \pm 0.16 & 2.41 & 1.03\end{array}$

0.65

0.80

0.67

0.42

0.72

0.81

0.95

0.96

0.97

0.86

0.99

1.03 
Aug. 2010 P20

$\begin{array}{cccccc}5 & 1.30 \pm 0.11 & 2.30 & 0.56 & - & - \\ 10 & 1.33 \pm 0.09 & 2.30 & 0.58 & - & - \\ 20 & 1.72 \pm 0.12 & 2.30 & 0.75 & - & - \\ 30 & 1.80 \pm 0.13 & 2.30 & 0.78 & 560 \pm 87 & - \\ 36 & 1.51 \pm 0.11 & 2.31 & 0.65 & - & - \\ 50 & 1.68 \pm 0.12 & 2.31 & 0.73 & 960 \pm 151 & - \\ 75 & 2.03 \pm 0.14 & 2.32 & 0.88 & - & - \\ 100 & 1.99 \pm 0.13 & 2.35 & 0.85 & 1521 \pm 338 & - \\ 150 & 2.20 \pm 0.14 & 2.39 & 0.92 & 1913 \pm 536 & - \\ 200 & 2.48 \pm 0.16 & 2.39 & 1.04 & 1982 \pm 758 & - \\ 300 & 2.33 \pm 0.16 & 2.40 & 0.97 & - & - \\ 500 & - & 2.41 & - & - & -\end{array}$

Aug. 2010 P26

$\begin{array}{cccccc}5 & 1.02 \pm 0.07 & 2.31 & 0.44 & - & - \\ 10 & 1.32 \pm 0.10 & 2.31 & 0.57 & - & - \\ 20 & 1.07 \pm 0.08 & 2.31 & 0.46 & - & - \\ 30 & 1.67 \pm 0.12 & 2.32 & 0.72 & 846 \pm 93 & - \\ 35 & 1.33 \pm 0.09 & 2.32 & 0.57 & - & - \\ 50 & 1.35 \pm 0.10 & 2.32 & 0.58 & 1386 \pm 139 & - \\ 75 & 1.83 \pm 0.12 & 2.32 & 0.79 & - & - \\ 100 & 2.08 \pm 0.14 & 2.33 & 0.89 & 2184 \pm 300 & - \\ 150 & 2.30 \pm 0.16 & 2.39 & 0.96 & 2429 \pm 518 & - \\ 200 & 2.27 \pm 0.15 & 2.39 & 0.95 & 2576 \pm 742 & - \\ 300 & 2.03 \pm 0.13 & 2.40 & 0.84 & - & - \\ 500 & 2.28 \pm 0.14 & 2.42 & 0.94 & - & -\end{array}$

Feb. 2011 P12

$\begin{array}{cccccc}1 & 1.96 \pm 0.13 & 2.29 & 0.86 & - & - \\ 5 & 2.13 \pm 0.14 & 2.29 & 0.93 & - & - \\ 10 & 2.18 \pm 0.15 & 2.29 & 0.95 & - & - \\ 20 & 2.12 \pm 0.14 & 2.29 & 0.93 & - & - \\ 30 & 2.22 \pm 0.15 & 2.29 & 0.97 & 234 \pm 171 & - \\ 50 & 2.01 \pm 0.13 & 2.29 & 0.88 & 337 \pm 234 & - \\ 75 & 2.25 \pm 0.15 & 2.29 & 0.98 & - & - \\ 100 & 2.35 \pm 0.15 & 2.35 & 1.00 & 468 \pm 426 & - \\ 150 & 2.51 \pm 0.16 & 2.39 & 1.05 & 381 \pm 648 & - \\ 200 & 2.44 \pm 0.16 & 2.40 & 1.02 & 265 \pm 878 & - \\ 300 & 2.35 \pm 0.15 & 2.40 & 0.98 & - & - \\ 500 & 2.37 \pm 0.15 & 2.41 & 0.98 & - & -\end{array}$

Feb. 2011 P16

$\begin{array}{cccc}1 & 1.87 \pm 0.13 & 2.31 & 0.81 \\ 10 & 2.05 \pm 0.13 & 2.31 & 0.89\end{array}$




$\begin{array}{cccccc}20 & 2.09 \pm 0.14 & 2.31 & 0.91 & - & - \\ 30 & 1.48 \pm 0.10 & 2.31 & 0.64 & 335 \pm 120 & - \\ 40 & 2.04 \pm 0.13 & 2.31 & 0.89 & - & - \\ 50 & 1.97 \pm 0.13 & 2.31 & 0.85 & 579 \pm 188 & - \\ 75 & 2.02 \pm 0.14 & 2.31 & 0.88 & - & - \\ 100 & 2.42 \pm 0.16 & 2.34 & 1.03 & 879 \pm 387 & - \\ 150 & 2.37 \pm 0.15 & 2.39 & 0.99 & 834 \pm 610 & - \\ 200 & 2.40 \pm 0.15 & 2.39 & 1.00 & 838 \pm 830 & - \\ 300 & 2.43 \pm 0.16 & 2.40 & 1.01 & - & - \\ 500 & 2.30 \pm 0.15 & 2.41 & 0.95 & - & -\end{array}$

Feb. 2011 P20

$\begin{array}{cccccc}1 & 2.12 \pm 0.14 & 2.30 & 0.92 & - & - \\ 10 & 2.06 \pm 0.14 & 2.30 & 0.90 & - & - \\ 20 & 1.99 \pm 0.13 & 2.30 & 0.87 & - & - \\ 30 & 2.07 \pm 0.14 & 2.30 & 0.90 & 215 \pm 120 & - \\ 35 & 2.05 \pm 0.14 & 2.30 & 0.89 & - & - \\ 50 & 2.03 \pm 0.14 & 2.30 & 0.88 & 360 \pm 196 & - \\ 75 & 1.95 \pm 0.13 & 2.30 & 0.85 & - & - \\ 100 & 2.47 \pm 0.16 & 2.35 & 1.05 & 661 \pm 394 & - \\ 150 & 2.46 \pm 0.16 & 2.39 & 1.03 & 526 \pm 624 & - \\ 200 & 2.55 \pm 0.17 & 2.39 & 1.07 & 366 \pm 860 & - \\ 300 & 2.27 \pm 0.15 & 2.40 & 0.95 & - & - \\ 500 & 2.35 \pm 0.16 & 2.41 & 0.97 & - & -\end{array}$

Feb. 2011 P26

\begin{tabular}{|c|c|c|c|c|c|}
\hline 5 & $2.21 \pm 0.14$ & 2.31 & 0.95 & - & - \\
\hline 10 & $2.31 \pm 0.16$ & 2.31 & 1.00 & - & - \\
\hline 25 & $2.14 \pm 0.15$ & 2.31 & 0.93 & - & - \\
\hline 30 & - & - & - & $114 \pm 132$ & - \\
\hline 50 & $2.30 \pm 0.15$ & 2.31 & 0.99 & $160 \pm 211$ & - \\
\hline 75 & $2.31 \pm 0.15$ & 2.31 & 1.00 & - & - \\
\hline 100 & $2.19 \pm 0.15$ & 2.36 & 0.93 & $224 \pm 418$ & - \\
\hline 125 & $2.44 \pm 0.15$ & 2.38 & 1.03 & - & - \\
\hline 150 & $2.27 \pm 0.15$ & 2.39 & 0.95 & $280 \pm 636$ & - \\
\hline 175 & $2.07 \pm 0.13$ & 2.39 & 0.87 & - & - \\
\hline 200 & $2.10 \pm 0.14$ & 2.39 & 0.88 & $659 \pm 836$ & - \\
\hline 250 & $2.06 \pm 0.13$ & 2.40 & 0.86 & - & - \\
\hline 300 & $2.15 \pm 0.16$ & 2.40 & 0.90 & - & - \\
\hline \multicolumn{6}{|c|}{ June 2011 P4 } \\
\hline 1 & $1.21 \pm 0.09$ & 2.25 & 0.54 & - & - \\
\hline 10 & $1.15 \pm 0.08$ & 2.25 & 0.51 & - & - \\
\hline 20 & $1.37 \pm 0.10$ & 2.28 & 0.60 & - & - \\
\hline 30 & $1.61 \pm 0.11$ & 2.29 & 0.70 & $849 \pm 91$ & - \\
\hline
\end{tabular}




$\begin{array}{cccccc}39 & 1.61 \pm 0.11 & 2.30 & 0.70 & - & - \\ 50 & 1.70 \pm 0.12 & 2.30 & 0.74 & 1232 \pm 149 & - \\ 75 & 2.27 \pm 0.15 & 2.32 & 0.98 & - & - \\ 100 & 2.11 \pm 0.14 & 2.36 & 0.89 & 1571 \pm 341 & - \\ 150 & 2.16 \pm 0.14 & 2.39 & 0.90 & 1921 \pm 538 & - \\ 200 & 2.21 \pm 0.14 & 2.40 & 0.92 & 2227 \pm 741 & - \\ 300 & 2.32 \pm 0.15 & 2.41 & 0.96 & - & - \\ 500 & 2.33 \pm 0.16 & 2.42 & 0.96 & - & -\end{array}$

June 2011 P12

$\begin{array}{cccccc}1 & 1.22 \pm 0.09 & 2.28 & 0.53 & - & - \\ 10 & 0.98 \pm 0.07 & 2.29 & 0.43 & - & - \\ 20 & 1.30 \pm 0.09 & 2.29 & 0.57 & - & - \\ 30 & 1.15 \pm 0.08 & 2.30 & 0.50 & 899 \pm 75 & - \\ 45 & 1.38 \pm 0.09 & 2.30 & 0.60 & - & - \\ 50 & 1.82 \pm 0.12 & 2.30 & 0.79 & 1450 \pm 126 & - \\ 75 & 2.51 \pm 0.16 & 2.32 & 1.08 & - & - \\ 100 & 2.46 \pm 0.15 & 2.37 & 1.04 & 1456 \pm 336 & - \\ 150 & 2.34 \pm 0.15 & 2.39 & 0.98 & 1425 \pm 550 & - \\ 200 & 2.16 \pm 0.14 & 2.40 & 0.90 & 1631 \pm 757 & - \\ 300 & 2.37 \pm 0.15 & 2.40 & 0.99 & - & - \\ 500 & 2.24 \pm 0.15 & 2.42 & 0.93 & - & -\end{array}$

June 2011 P16

\begin{tabular}{|c|c|c|c|c|c|}
\hline 1 & $1.69 \pm 0.11$ & 2.31 & 0.73 & - & - \\
\hline 10 & $1.56 \pm 0.11$ & 2.31 & 0.67 & - & - \\
\hline 20 & $1.86 \pm 0.12$ & 2.31 & 0.80 & - & 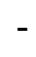 \\
\hline 30 & $1.04 \pm 0.08$ & 2.31 & 0.45 & $544 \pm 95$ & 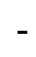 \\
\hline 50 & $1.40 \pm 0.09$ & 2.31 & 0.61 & $1172 \pm 141$ & - \\
\hline 60 & $1.79 \pm 0.12$ & 2.31 & 0.77 & - & - \\
\hline 75 & $1.82 \pm 0.12$ & 2.31 & 0.79 & - & - \\
\hline 100 & $2.50 \pm 0.16$ & 2.33 & 1.07 & $1715 \pm 321$ & - \\
\hline 150 & $2.56 \pm 0.16$ & 2.39 & 1.07 & $1474 \pm 553$ & - \\
\hline 200 & $2.27 \pm 0.14$ & 2.39 & 0.95 & $1447 \pm 774$ & - \\
\hline 300 & $2.20 \pm 0.14$ & 2.40 & 0.92 & - & - \\
\hline 500 & - & 2.41 & - & - & - \\
\hline \multicolumn{6}{|c|}{ June 2011 P20 } \\
\hline 1 & $1.56 \pm 0.11$ & 2.30 & 0.68 & - & - \\
\hline 10 & $1.55 \pm 0.10$ & 2.30 & 0.67 & - & - \\
\hline 20 & $1.34 \pm 0.09$ & 2.30 & 0.58 & - & - \\
\hline 30 & $1.50 \pm 0.10$ & 2.30 & 0.65 & $746 \pm 86$ & - \\
\hline 50 & $1.48 \pm 0.10$ & 2.30 & 0.64 & $1217 \pm 143$ & - \\
\hline 55 & $1.56 \pm 0.11$ & 2.30 & 0.68 & - & - \\
\hline 75 & $2.24 \pm 0.15$ & 2.31 & 0.97 & - & - \\
\hline
\end{tabular}




$\begin{array}{cccccc}100 & 2.54 \pm 0.16 & 2.34 & 1.09 & 1525 \pm 340 & - \\ 150 & 2.47 \pm 0.16 & 2.39 & 1.03 & 1335 \pm 569 & - \\ 200 & 2.20 \pm 0.14 & 2.39 & 0.92 & 1424 \pm 785 & - \\ 300 & 2.32 \pm 0.15 & 2.40 & 0.97 & - & - \\ 500 & 2.39 \pm 0.16 & 2.42 & 0.99 & - & -\end{array}$

June 2011 P26 D (Trap Depolyment)

$\begin{array}{cccccc}1 & 1.17 \pm 0.08 & 2.31 & 0.51 & - & - \\ 10 & 1.30 \pm 0.08 & 2.31 & 0.56 & - & - \\ 20 & 1.20 \pm 0.08 & 2.31 & 0.52 & - & - \\ 30 & 1.27 \pm 0.09 & 2.31 & 0.55 & 949 \pm 78 & 3192 \pm 117 \\ 45 & 1.38 \pm 0.09 & 2.31 & 0.60 & - & - \\ 50 & 1.35 \pm 0.09 & 2.32 & 0.58 & 1514 \pm 126 & 2909 \pm 92 \\ 75 & 2.12 \pm 0.13 & 2.32 & 0.91 & - & - \\ 83 & - & - & - & 1974 \pm 235 & - \\ 100 & 2.24 \pm 0.14 & 2.32 & 0.96 & 2033 \pm 301 & 2256 \pm 94 \\ 150 & 2.49 \pm 0.16 & 2.39 & 1.04 & 2021 \pm 514 & 1928 \pm 79 \\ 200 & 2.51 \pm 0.16 & 2.39 & 1.05 & 1867 \pm 739 & 2281 \pm 97 \\ 300 & 2.28 \pm 0.14 & 2.40 & 0.95 & - & - \\ 500 & 2.30 \pm 0.14 & 2.42 & 0.95 & - & -\end{array}$

June 2011 P26 R (Trap Recovery)

$\begin{array}{cccccc}1 & 1.22 \pm 0.08 & 2.32 & 0.53 & - & - \\ 10 & 1.35 \pm 0.09 & 2.32 & 0.58 & - & - \\ 20 & 1.20 \pm 0.08 & 2.32 & 0.52 & - & - \\ 30 & 1.28 \pm 0.08 & 2.32 & 0.55 & 923 \pm 78 & 3192 \pm 117 \\ 48 & 1.68 \pm 0.11 & 2.32 & 0.73 & - & - \\ 50 & 1.59 \pm 0.10 & 2.32 & 0.68 & 1395 \pm 130 & 2909 \pm 92 \\ 75 & 2.01 \pm 0.12 & 2.32 & 0.87 & - & - \\ 85 & - & - & - & 1847 \pm 246 & - \\ 100 & 2.25 \pm 0.13 & 2.32 & 0.97 & 1910 \pm 301 & 2256 \text { v } 94 \\ 150 & 2.47 \pm 0.14 & 2.36 & 1.05 & 1881 \pm 499 & 1928 \pm 79 \\ 200 & 2.29 \pm 0.14 & 2.39 & 0.96 & 1871 \pm 705 & 2281 \pm 97 \\ 300 & 2.31 \pm 0.14 & 2.40 & 0.96 & - & - \\ 500 & 2.38 \pm 0.14 & 2.42 & 0.98 & - & -\end{array}$

Feb. 2012 P4

$\begin{array}{cccccc}1 & 1.79 \pm 0.12 & 2.30 & 0.78 & - & - \\ 10 & 1.74 \pm 0.11 & 2.30 & 0.76 & - & - \\ 20 & 1.94 \pm 0.13 & 2.30 & 0.85 & - & - \\ 30 & 1.91 \pm 0.13 & 2.30 & 0.83 & 336 \pm 135 & - \\ 40 & 1.74 \pm 0.11 & 2.30 & 0.76 & - & - \\ 50 & 2.09 \pm 0.13 & 2.30 & 0.91 & 581 \pm 193 & - \\ 75 & 2.00 \pm 0.13 & 2.31 & 0.87 & - & - \\ 100 & 1.99 \pm 0.13 & 2.37 & 0.84 & 1015 \pm 370 & \end{array}$




$\begin{array}{llllcl}150 & 2.23 \pm 0.14 & 2.39 & 0.93 & 1401 \pm 562 & - \\ 200 & 2.22 \pm 0.14 & 2.40 & 0.93 & 1646 \pm 763 & - \\ 300 & 2.40 \pm 0.15 & 2.40 & 1.00 & - & - \\ 500 & 2.28 \pm 0.15 & 2.42 & 0.94 & - & -\end{array}$

Feb. 2012 P12

$\begin{array}{cccccc}1 & 1.94 \pm 0.11 & 2.31 & 0.84 & - & - \\ 10 & 1.70 \pm 0.10 & 2.31 & 0.73 & - & - \\ 20 & 2.07 \pm 0.12 & 2.31 & 0.90 & - & - \\ 30 & 2.04 \pm 0.12 & 2.31 & 0.88 & 350 \pm 104 & - \\ 40 & - & 2.31 & - & - & - \\ 50 & 2.18 \pm 0.14 & 2.31 & 0.94 & 356 \pm 180 & - \\ 75 & 1.91 \pm 0.12 & 2.31 & 0.83 & - & - \\ 100 & 2.17 \pm 0.13 & 2.31 & 0.94 & 739 \pm 357 & - \\ 150 & 2.79 \pm 0.16 & 2.39 & 1.17 & 551 \pm 571 & - \\ 200 & 2.66 \pm 0.17 & 2.40 & 1.11 & 71 \pm 807 & - \\ 300 & 2.41 \pm 0.15 & 2.40 & 1.01 & - & - \\ 500 & 2.35 \pm 0.15 & 2.42 & 0.97 & - & -\end{array}$

Feb. 2012 P16

$\begin{array}{cccccc}1 & 1.76 \pm 0.11 & 2.31 & 0.76 & - & - \\ 10 & 1.64 \pm 0.11 & 2.31 & 0.71 & - & - \\ 20 & 1.81 \pm 0.11 & 2.31 & 0.78 & - & - \\ 30 & 1.81 \pm 0.11 & 2.31 & 0.78 & 435 \pm 105 & - \\ 40 & 1.83 \pm 0.11 & 2.31 & 0.79 & - & - \\ 50 & 1.80 \pm 0.11 & 2.31 & 0.78 & 719 \pm 165 & - \\ 75 & 2.24 \pm 0.14 & 2.31 & 0.97 & - & - \\ 100 & 2.40 \pm 0.14 & 2.31 & 1.04 & 922 \pm 352 & - \\ 150 & 2.71 \pm 0.16 & 2.40 & 1.13 & 632 \pm 567 & - \\ 200 & 2.83 \pm 0.17 & 2.40 & 1.18 & 96 \pm 799 & - \\ 300 & 2.73 \pm 0.16 & 2.40 & 1.14 & - & - \\ 500 & 2.75 \pm 0.15 & 2.41 & 1.14 & - & -\end{array}$

Feb. 2012 P20

$\begin{array}{cccccc}1 & 2.16 \pm 0.13 & 2.31 & 0.93 & - & - \\ 10 & 2.06 \pm 0.12 & 2.31 & 0.89 & - & - \\ 20 & 2.04 \pm 0.12 & 2.31 & 0.88 & - & - \\ 30 & 2.03 \pm 0.12 & 2.31 & 0.88 & 121 \pm 123 & - \\ 40 & 2.27 \pm 0.14 & 2.31 & 0.98 & - & - \\ 50 & 1.98 \pm 0.12 & 2.31 & 0.86 & 220 \pm 188 & - \\ 75 & 2.13 \pm 0.12 & 2.31 & 0.92 & - & - \\ 100 & 1.97 \pm 0.12 & 2.31 & 0.85 & 593 \pm 357 & - \\ 150 & 2.68 \pm 0.16 & 2.40 & 1.12 & 636 \pm 555 & - \\ 200 & 2.23 \pm 0.14 & 2.40 & 0.93 & 545 \pm 765 & - \\ 300 & 2.35 \pm 0.14 & 2.40 & 0.98 & - & -\end{array}$


$\begin{array}{llll}500 & 2.45 \pm 0.14 & 2.41 & 1.02\end{array}$

Feb. 2012 P26

$\begin{array}{cccccc}1 & 1.90 \pm 0.12 & 2.31 & 0.82 & - & - \\ 10 & 2.33 \pm 0.14 & 2.31 & 1.01 & - & - \\ 20 & 2.12 \pm 0.12 & 2.31 & 0.92 & - & - \\ 30 & 2.23 \pm 0.13 & 2.31 & 0.96 & 78 \pm 125 & - \\ 50 & 2.31 \pm 0.14 & 2.31 & 1.00 & 105 \pm 194 & - \\ 75 & 2.07 \pm 0.12 & 2.31 & 0.90 & 195 \pm 283 & - \\ 100 & 2.03 \pm 0.12 & 2.31 & 0.88 & 386 \pm 369 & - \\ 125 & 1.65 \pm 0.10 & 2.32 & 0.71 & - & - \\ 150 & 2.55 \pm 0.14 & 2.37 & 1.08 & 898 \pm 534 & - \\ 200 & 2.49 \pm 0.15 & 2.39 & 1.04 & 694 \pm 741 & - \\ 300 & 2.56 \pm 0.15 & 2.40 & 1.07 & - & - \\ 500 & 2.58 \pm 0.15 & 2.41 & 1.07 & - & -\end{array}$

June 2012 P4

$\begin{array}{cccccc}1 & 0.84 \pm 0.06 & 2.25 & 0.38 & - & - \\ 10 & 0.82 \pm 0.06 & 2.25 & 0.36 & - & - \\ 20 & 0.91 \pm 0.07 & 2.25 & 0.40 & - & - \\ 30 & 1.70 \pm 0.12 & 2.28 & 0.75 & 907 \pm 91 & - \\ 40 & 1.99 \pm 0.14 & 2.30 & 0.86 & - & - \\ 50 & 1.88 \pm 0.13 & 2.31 & 0.82 & 1143 \pm 155 & - \\ 75 & 2.37 \pm 0.16 & 2.31 & 1.03 & - & - \\ 100 & 1.91 \pm 0.13 & 2.34 & 0.81 & 1408 \pm 359 & - \\ 150 & 2.49 \pm 0.16 & 2.39 & 1.04 & 1649 \pm 565 & - \\ 200 & 2.54 \pm 0.17 & 2.40 & 1.06 & 1474 \pm 798 & - \\ 300 & 2.26 \pm 0.15 & 2.41 & 0.94 & - & - \\ 500 & 2.49 \pm 0.16 & 2.42 & 1.03 & - & -\end{array}$

June 2012 P12

$\begin{array}{cccccc}1 & 1.26 \pm 0.08 & 2.31 & 0.54 & - & - \\ 10 & 1.38 \pm 0.09 & 2.31 & 0.60 & - & - \\ 20 & 1.31 \pm 0.09 & 2.31 & 0.57 & - & - \\ 30 & 1.73 \pm 0.12 & 2.31 & 0.75 & 678 \pm 90 & - \\ 50 & 2.03 \pm 0.14 & 2.31 & 0.88 & 928 \pm 161 & - \\ 60 & 2.02 \pm 0.13 & 2.31 & 0.87 & - & - \\ 75 & 2.33 \pm 0.15 & 2.31 & 1.01 & - & - \\ 100 & 2.55 \pm 0.17 & 2.32 & 1.10 & 982 \pm 373 & - \\ 150 & 2.02 \pm 0.14 & 2.39 & 0.85 & 1085 \pm 591 & - \\ 164 & - & - & - & 1209 \pm 647 & - \\ 200 & 2.46 \pm 0.16 & 2.40 & 1.03 & 1305 \pm 805 & - \\ 300 & 2.36 \pm 0.16 & 2.40 & 0.98 & - & - \\ 500 & 2.36 \pm 0.16 & 2.41 & 0.98 & - & -\end{array}$

June 2012 P16 


$\begin{array}{cccccc}1 & 1.62 \pm 0.12 & 2.32 & 0.70 & - & - \\ 10 & 1.55 \pm 0.11 & 2.32 & 0.67 & - & - \\ 20 & 1.38 \pm 0.10 & 2.32 & 0.60 & - & - \\ 30 & 1.55 \pm 0.11 & 2.32 & 0.67 & 637 \pm 95 & - \\ 40 & 1.67 \pm 0.12 & 2.32 & 0.72 & - & - \\ 50 & 1.80 \pm 0.13 & 2.32 & 0.77 & 1008 \pm 160 & - \\ 75 & 2.21 \pm 0.15 & 2.32 & 0.95 & - & - \\ 100 & 2.33 \pm 0.16 & 2.32 & 1.00 & 1270 \pm 368 & - \\ 150 & 2.58 \pm 0.17 & 2.40 & 1.08 & 1128 \pm 603 & - \\ 200 & 2.44 \pm 0.16 & 2.40 & 1.02 & 960 \pm 842 & - \\ 300 & 2.37 \pm 0.16 & 2.40 & 0.99 & - & - \\ 500 & 2.49 \pm 0.16 & 2.41 & 1.03 & - & -\end{array}$

June 2012 P20

$\begin{array}{cccccc}1 & 1.61 \pm 0.11 & 2.31 & 0.70 & - & - \\ 10 & 1.57 \pm 0.11 & 2.31 & 0.68 & - & - \\ 20 & 1.62 \pm 0.11 & 2.31 & 0.70 & - & - \\ 30 & 1.83 \pm 0.13 & 2.31 & 0.79 & 551 \pm 95 & - \\ 44 & 1.62 \pm 0.11 & 2.31 & 0.70 & - & - \\ 50 & 2.36 \pm 0.16 & 2.32 & 1.02 & 843 \pm 167 & - \\ 75 & 2.12 \pm 0.14 & 2.32 & 0.92 & - & - \\ 100 & 2.68 \pm 0.17 & 2.32 & 1.15 & 841 \pm 385 & - \\ 115 & - & - & - & 699 \pm 458 & - \\ 150 & 2.57 \pm 0.17 & 2.39 & 1.08 & 454 \pm 629 & - \\ 200 & - & 2.40 & - & 192 \pm 873 & - \\ 300 & - & 2.40 & - & - & - \\ 500 & - & 2.41 & - & - & \end{array}$

June 2012 P26 D (Trap Deployment)

$\begin{array}{cccccc}1 & 1.64 \pm 0.11 & 2.32 & 0.71 & - & \\ 10 & 1.95 \pm 0.13 & 2.32 & 0.84 & - & \\ 20 & 1.51 \pm 0.11 & 2.32 & 0.65 & - & \\ 30 & 1.59 \pm 0.11 & 2.32 & 0.69 & 513 \pm 104 & 3999 \pm 206 \\ 40 & 1.43 \pm 0.10 & 2.32 & 0.62 & - & - \\ 50 & 1.39 \pm 0.10 & 2.32 & 0.60 & 1008 \pm 161 & 5485 \pm 290 \\ 60 & - & - & - & 1241 \pm 190 & - \\ 75 & 1.98 \pm 0.13 & 2.32 & 0.85 & - & - \\ 100 & 2.12 \pm 0.15 & 2.32 & 0.92 & 1655 \pm 342 & 3154 \pm 192 \\ 150 & 2.21 \pm 0.15 & 2.38 & 0.93 & 1918 \pm 551 & 2151 \pm 135 \\ 200 & 2.19 \pm 0.14 & 2.39 & 0.91 & 2186 \pm 760 & 3959 \pm 129 \\ 300 & 2.39 \pm 0.16 & 2.40 & 1.00 & - & - \\ 500 & 2.51 \pm 0.17 & 2.42 & 1.04 & - & -\end{array}$

June 2012 P26 R (Trap Recovery)

$\begin{array}{llll}1 & 1.34 \pm 0.10 & 2.32 & 0.58\end{array}$ 


\begin{tabular}{cccccc}
10 & $1.38 \pm 0.10$ & 2.32 & 0.60 & - & - \\
20 & $1.60 \pm 0.11$ & 2.32 & 0.69 & - & - \\
30 & $1.71 \pm 0.11$ & 2.32 & 0.74 & $706 \pm 94$ & $3999 \pm 206$ \\
40 & $1.71 \pm 0.12$ & 2.32 & 0.74 & - & - \\
50 & $1.62 \pm 0.11$ & 2.32 & 0.70 & $1068 \pm 157$ & $5485 \pm 290$ \\
60 & - & - & - & $1249 \pm 188$ & - \\
75 & $1.95 \pm 0.13$ & 2.32 & 0.84 & - & - \\
100 & $2.27 \pm 0.14$ & 2.32 & 0.98 & $1599 \pm 338$ & $3154 \pm 192$ \\
150 & $2.41 \pm 0.16$ & 2.38 & 1.01 & $1617 \pm 553$ & $2151 \pm 135$ \\
200 & $2.40 \pm 0.16$ & 2.39 & 1.01 & $1589 \pm 780$ & $3959 \pm 129$ \\
300 & $2.33 \pm 0.15$ & 2.40 & 0.97 & - & - \\
500 & $2.38 \pm 0.16$ & 2.42 & 0.98 & - & - \\
\hline
\end{tabular}




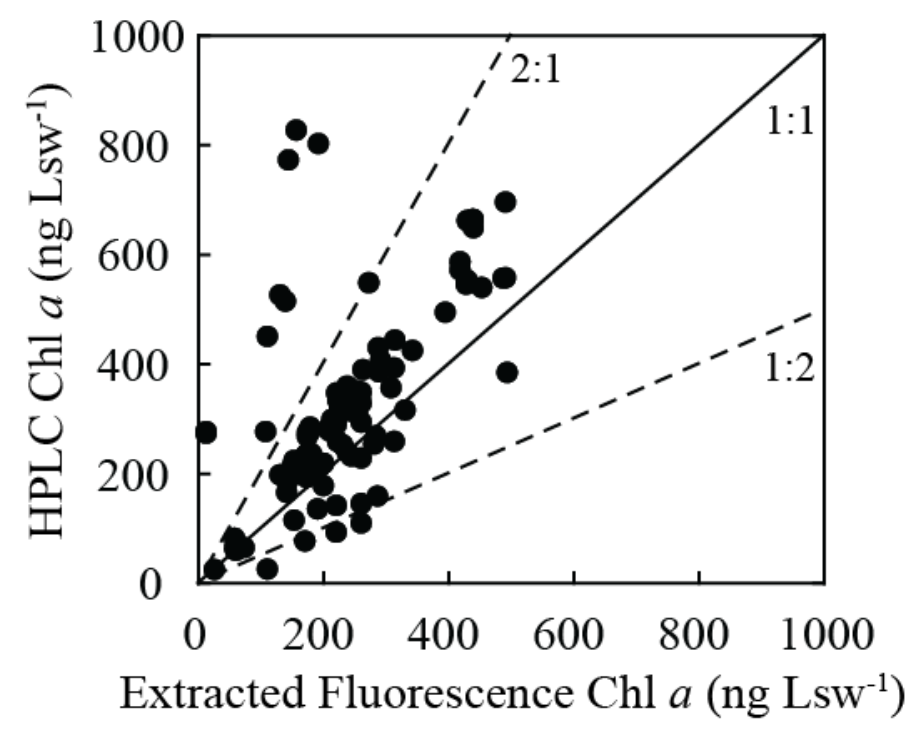

Figure S1. Comparison of fluorescence and high-performance liquid chromatography (HPLC) measurements of $\mathrm{Chl} a$ concentrations. 


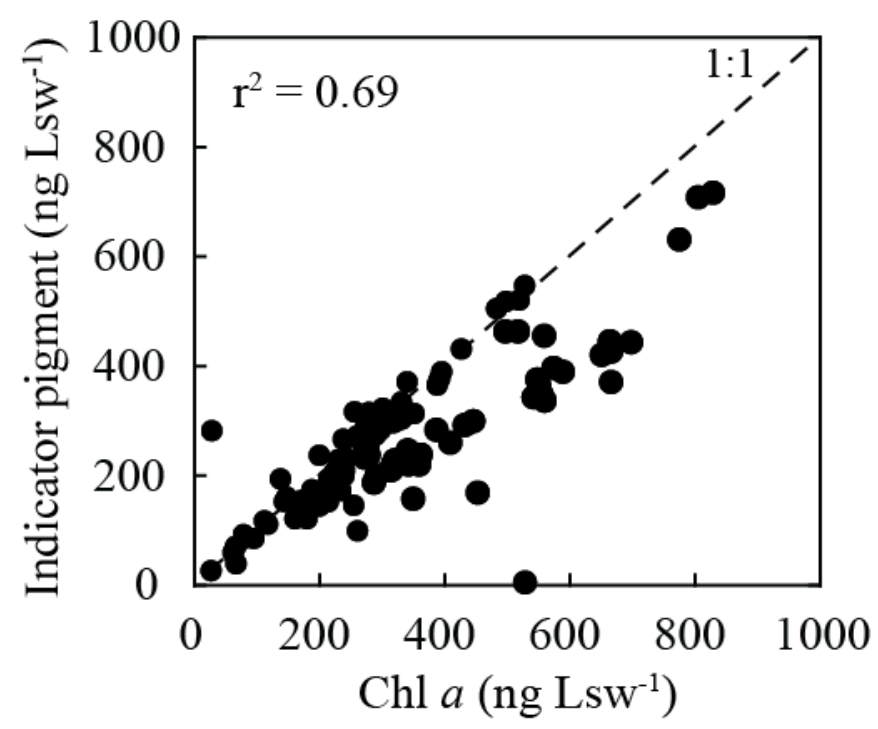

Figure S2. Chl $a$ concentrations plotted against total indicator pigment concentrations for Niskin bottle samples. The correlation coefficient $\left(\mathrm{r}^{2}\right)$ is also shown. 


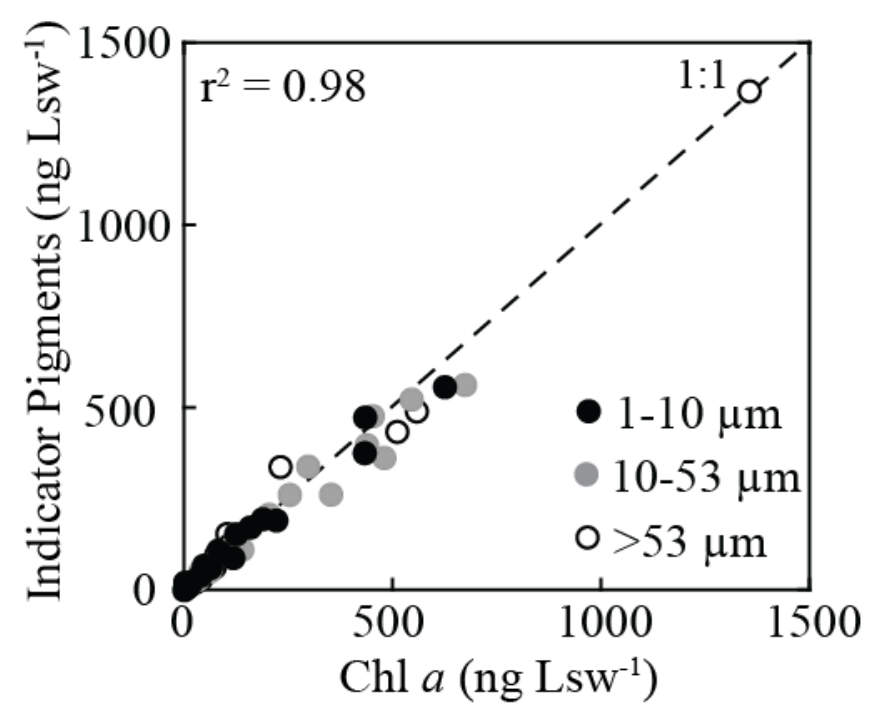

Figure S3. Chl $a$ concentrations plotted against total indicator pigment concentrations for three size-fractions of particles collected by in situ pumps. The correlation coefficient $\left(r^{2}\right)$ is also shown. 


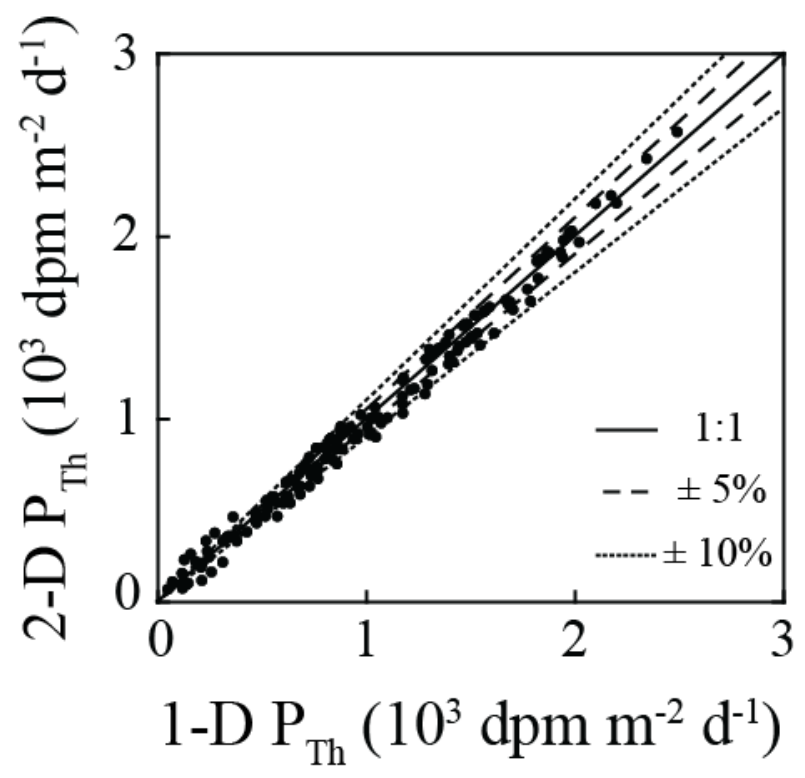

Figure S4. ${ }^{234}$ Th fluxes determined by the 1-D steady-state model compared to those determined by the 2-D steady-state model. Dashed and dotted lines envelope 5\% and $10 \%$ error ranges respectively. 